\title{
不同叶龄藮、穗氮肥组合对粳稻产量及氮素利用的影响
}

\author{
王艳易 军 高继平 ${ }^{*}$ 张丽娜杨继芬赵艳泽辛威 \\ 甄晓溪 张文忠 ${ }^{*}$
}

沈阳农业大学水稻研究所 / 教育部省部共建北方粳稻遗传育种重点实验室 / 农业农村部东北水稻生物学与遗传育种重点实验室, 辽宁沈阳 110161

摘 要：以主茎叶片数不同的粳稻品种吉粳 88 (14 片)、沈农 265 (15 片)和沈农 1401 (16 片)为试材, 采用大田筒栽方 式, 在总施氮量 $225 \mathrm{~kg} \mathrm{hm}^{-2}$ 及轻简施肥(基肥、藍肥、穗肥)模式基础上, 设置基藍肥：穗肥 6: 4 和 8: 2 两种施肥 比例, 并分设不同源、库叶龄期施氮组合即不同叶龄菜、穗肥精确施氮组合。分析了不同源库期氮肥运筹模式对水 稻农艺性状、产量及氮素利用特性的影响。结果表明: (1)在有效穗数、分化颖花数、产量和氮素利用率方面, 吉粳 88 、沈农 265 、沈农 1401 不同氮肥运筹下最佳菜、穗肥叶龄组合均为 $6: 4$ 显著高于 8：2。(2)不同氮肥运筹下，吉 粳 88 在 8 叶(叶龄指数 $57.1 \%$ )、沈农 265 在 9 叶(叶龄指数 $60.0 \%$ )、沈农 1401 在 10 叶(叶龄指数 $62.5 \%$ )时, 即叶龄 指数在 $60 \%$ 左右时, 施用蒒莘肥效果最佳, 最终穗数最多, 对保臨起主要作用; 吉粳 88 在 11 叶(叶龄指数 $78.6 \%$ )、沈 农 265 在 12 叶(叶龄指数 $80.0 \%$ )、沈农 1401 在 13 叶(叶龄指数 $81.3 \%$ )时, 即叶龄指数在 $80 \%$ 左右时, 施用穗肥效果 最佳, 最终穗粒数最多, 对促花起主要作用。(3)吉粳 88-6：4 (8,11), 沈农 265-6：4 (9, 12), 沈农 1401-6: 4 (10,13) 3 组处理, 在产量、氮素积累量、氮素吸收利用率、农学利用率及偏生产力等方面, 显著高于同品种不同叶龄櫱、穗氮 肥组合中的其他处理。因此, 适当延迟藍肥施用叶龄期(叶龄指数 $60 \%$ 左右)、提前穗肥施用叶龄期(叶龄指数 $80 \%$ 左 右)同时增加穗肥施用比例, 既可以显著提高氮素积累量、氮素吸收利用率、农学利用率及偏生产力, 又能显著促进 成穗率的提高和颖花数的分化, 达到保藍促花的双重作用, 实现优源、扩库、充实的目标, 从而获得高产。

关键词：粳稻; 保藍氮肥; 促花氮肥; 产量; 氮素利用效率

\section{Effects of precision leaf age fertilization on yield and nitrogen utilization of japonica rice}

WANG Yan, YI Jun, GAO Ji-Ping ${ }^{*}$, ZHANG Li-Na, YANG Ji-Fen, ZHAO Yan-Ze, XIN Wei, ZHEN Xiao-Xi, and ZHANG Wen-Zhong ${ }^{*}$

Rice Research Institute, Shenyang Agricultural University / Key Laboratory of Northern Japonica Rice Genetics and Breeding, Ministry of Education and Liaoning Province / Key Laboratory of Northeast Rice Biology, Genetics and Breeding, Ministry of Agriculture and Rural Affairs, Shenyang 110161, Liaoning, China

Abstract: The japonica rice cultivar Jigeng 88 (14 leaves), Shennong 265 (15 leaves) and Shennong 1401 (16 leaves) with different main leaf ages were used to analyze the effects of different source-sink nitrogen fertilizer application models on agronomic traits, yield and nitrogen utilization characteristics of rice grown in the field-tube condition. Two kinds of fertilizer proportions (base fertilizer: panicle fertilizer was 6:4 and 8:2) were set under $225 \mathrm{~kg} \mathrm{hm}^{-2}$ of nitrogen application based on the light and simple fertilization model (basal fertilizer, tillering fertilizer, panicle fertilizer). The tillering fertilizer was applied at the stage of

\footnotetext{
本研究由国家重点研发计划项目(2018YFD0300306), 国家自然科学基金项目(31501250), 辽宁省百千万人才工程项目(2015-39)和沈阳 市科技计划项目(17-231-37)资助。

This study was financed by the National Key Research and Development Program (2018YFD0300306), the National Natural Science Foundation of China (31501250), the Liaoning Bai-Qian-Wan Talents Program (2015-39), and the Project of Shenyang Science and Technology (17-231-37).

*通信作者(Corresponding authors): 高继平, E-mail: gaojiping1983@163.com; 张文忠, E-mail: zwzhong@126.com

第一作者联系方式: E-mail: 2422437380@qq.com

Received (收稿日期): 2019-03-01; Accepted (接受日期): 2019-08-09; Published online (网络出版日期): 2019-09-10.

URL: http://kns.cnki.net/kcms/detail/11.1809.S.20190910.1132.008.html
} 
source construction, and panicle fertilizer was applied at stage of sink formation. The precise nitrogen application combined different nitrogen fertilizer modes for tillering and panicle fertilizers with different leaf ages. Number of differentiated spikelets, effective panicles, yield, and nitrogen use efficiency, were significantly higher by using the 6:4 fertilizer proportions than by using 8:2 under all nitrogen fertilizer application models in these cultivars. Under different nitrogen fertilizer regimes, the 8 leaves of Jigeng 88 (leaf age index was 57.1\%), 9 leaves of Shennong 265 (leaf age index was 60.0\%), and 10 leaves of Shennong 1401 (leaf age index was $62.55 \%$ ) were the best stage to apply tillering fertilizer. It means that the leaf age index about $60 \%$ is better to protect the more panicle formation. The 11 leaves of Jigeng 88 (leaf age index was 78.6\%), 12 leaves of Shennong 265 (leaf age index was $80.0 \%$ ), 13 leaves of Shennong 1401 (leaf age index was $81.3 \%$ ) were the best stage to apply panicle fertilizer. It means that leaf age index about $80 \%$ is better to promote spikelet differentiation. The yield, nitrogen accumulation, nitrogen uptake and utilization, agronomic efficiency, and partial productivity were significantly higher in $6: 4(8,11)$ of Jigeng $88,6: 4(9,12)$ of Shennong 265, 6:4 $(10,13)$ of Shennong 1401 than in other treatments. Therefore, the delayed leaf age of tillering nitrogen fertilizer application (leaf age index was about 60\%), ahead of applied the panicle fertilizer (leaf age index was about $80 \%$ ), and increasing the ratio of nitrogen fertilizer application not only significantly increase nitrogen accumulation, nitrogen absorption and utilization, nitrogen agronomic utilization rate and partial productivity but also promote number of differentiated spikelets and the percentage of effective panicle number, achieving the dual role of protecting and promoting spikelets, and high yields.

Keywords: japonica rice; tillering protecting nitrogen fertilizer; flower promoting nitrogen fertilizer; yield; nitrogen use efficiency

氮肥运筹是水稻生产过程中最重要的营养调 控手段之一, 科学的氮肥运筹对提高水稻氮素吸收 利用率, 增加水稻产量及提升品质至关重要。关于 氮肥的合理运筹, 前人在施用总量、前氮后移、基 藮肥与穗肥比例、施用次数及施用时期对水稻群体 指标、产量和氮素利用率的影响等方面做了大量研 究 $^{[1-9]}$, 一致认为提高水稻生育后期氮肥施用比例可 显著优化群体质量、提高产量和氮素利用率, 并在 此基础上形成了一批氮肥运筹技术(“高产高效栽培 技术”、“精确定量栽培技术”、“三段五次”、“稳前、

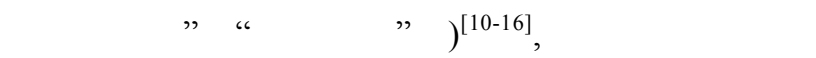
肥施用模式起到了重要作用。纵观以往的研究发现, 由于水稻种植的生态区域或研究所选用的水稻品种 类型不同, 其研究结果具有一定局限性。目前关于 不同叶龄藍、穗氮肥组合对东北不同生育期高产粳 稻品种产量及氮素利用的影响鲜有报道。本研究以 叶龄模式为基础, 采用 3 个不同主茎叶片数的粳稻 品种, 设置不同基臨肥与穗肥比例, 按照“源”建成 期叶龄和“库”建成期叶龄逐叶进行精确组合施氮, 剖析不同藍、穗肥组合对水稻形态建成、产量构成 以及氮素利用的利弊影响, 明确不同粳稻品种适宜 的菜、穗肥施用时期, 为系统构建及应用高产高效 精确施氮模式奠定基础。

\section{1 材料与方法}

\section{1 试验地点与供试品种}

试验于2016-2017年在沈阳农业大学水稻研究 所试验基地进行 $\left(41^{\circ} 50^{\prime} \mathrm{N}, 123^{\circ} 24^{\prime} \mathrm{E}\right)$ 。2年试验水稻 生长季节的温度、降雨量、湿度、日照时数等气象
数据见表1 (来源于国家气象数据中心)。土壤类型为 沙壤土, 地力中等偏上。2016年耕层 $(0 \sim 20 \mathrm{~cm})$ 含全氮 $0.92 \mathrm{~g} \mathrm{~kg}^{-1}$ 、有机质 $16.25 \mathrm{~g} \mathrm{~kg}^{-1}$ 、速效磷 $70.55 \mathrm{mg} \mathrm{kg}^{-1}$ 、 速效钾115.04 mg kg-1 $\mathrm{pH} 7.2$ 。2017年耕层 $(0 \sim 20 \mathrm{~cm})$ 含全 氮 $0.89 \mathrm{~g} \mathrm{~kg}^{-1}$ 、有机质 $17.30 \mathrm{~g} \mathrm{~kg}^{-1}$ 、速效磷70.56 $\mathrm{mg} \mathrm{kg}^{-1}$ 、 速效钾115.06 $\mathrm{mg} \mathrm{kg}^{-1}, \mathrm{pH} 7.1$ 。

供试品种吉粳 88 (吉林省超级稻品种), 主茎叶 片数 14 , 伸长节间数 5 , 全生育期 $145 \mathrm{~d}$ 左右; 沈农 265 (辽宁省超级稻品种), 主茎叶片数 15, 伸长节间 数 5 , 全生育期 $157 \mathrm{~d}$ 左右; 沈农 1401 (辽宁省超高 产品系), 主茎叶片数 16 , 伸长节间数 5 , 全生育期 $160 \mathrm{~d}$ 左右。

\section{2 田间试验设计}

全生育期施尿素(N 46\%) $489.1 \mathrm{~kg} \mathrm{hm}^{-2}$ (纯氮量 $225 \mathrm{~kg} \mathrm{hm}^{-2}$ )、过磷酸钙 (含 $\mathrm{P}_{2} \mathrm{O}_{5} 12 \%$ ) $937.5 \mathrm{~kg} \mathrm{hm}^{-2}$ 、 硫酸钾(含 $\mathrm{K}_{2} \mathrm{O} 50 \%$ ) $225.0 \mathrm{~kg} \mathrm{hm}^{-2}, \mathrm{~N}: \mathrm{P}_{2} \mathrm{O}_{5}$ ： $\mathrm{K}_{2} \mathrm{O}$ 比例为 $2: 1: 1$ 。采用水溶法, 磷肥作基肥一次性全 量施入, 钾肥分基肥(移栽前 1 d)和穗肥(倒4叶)两次 等量施入。氮肥设置2个运筹比例, 即基蒒肥与穗肥 比例分别为 $6: 4$ 和 $8: 2$, 基肥与分葟肥中的氮肥施 用比例为 $6: 4$ (表2), 另设不施氮空白组(N0), 与此 同时设置菜肥与穗肥施用叶龄期组合处理试验, 其 中菜肥设置3个施用叶龄时期(吉粳 88 为 $6 、 7 、 8$ 叶, 沈 农 265 为 $7 、 8 、 9$ 叶, 沈农 1401 为 $8 、 9 、 10$ 叶), 穗肥 设置2个施用叶龄时期, 即苞分化期(倒4叶)和颖花 分化期(倒 2 叶), 即吉粳 88 为 11 叶和 13 叶, 沈农 265 为 12 叶和14叶, 沈农 1401 为 13 叶和 15 叶(表3), 共39个 处理, 每处理 1 筒, 重复3次, 总计 117 筒。在耙地均 匀后埋设内径为 $30 \mathrm{~cm}$ 的无底“315PVC 平壁圆筒”, 
壁厚 $0.5 \mathrm{~cm}$, 筒高 $35 \mathrm{~cm}$, 将筒钉压至犁底层 $(25 \mathrm{~cm}$ 处)。基施磷、钾肥在钉筒前水耙地时统一进行, 基 施氮肥(尿素)由于各处理不同，在钉筒后分筒单施。 采用旱育积塑料硬盘方式培育秧苗，于4月21日播 种，5月24日移栽(移栽叶龄5.0叶)，每筒2穴，每穴2
株苗, 穴距 $15.5 \mathrm{~cm}$, 南北行向, 行距 $31 \mathrm{~cm}$ (筒直径 + 壁厚)，各筒在水稻生长期按表3确定的叶片组合施 肥，单独灌水。将所有筒连排紧靠形成一个长 $8.40 \mathrm{~m}$ 、宽 $4.42 \mathrm{~m}$ 的田间小区, 筒外种植普通品种作 为保护行。

\section{表 1 水稻生育期间温度、降雨量、相对湿度和日照时数}

Table 1 Monthly temperature, rainfall, relative humidity, and sunshine hours during the growth season in 2016 and 2017

\begin{tabular}{|c|c|c|c|c|c|c|c|c|c|c|}
\hline \multirow[t]{2}{*}{$\begin{array}{c}\text { 月份 } \\
\text { Month }\end{array}$} & $\begin{array}{r}\text { 平均 } \\
\text { 最 } \\
\text { Mir }\end{array}$ & $\begin{array}{l}\text { 直 Aver } \\
\text { um }\end{array}$ & $\begin{array}{r}\text { temper } \\
\text { 最 } \\
\mathrm{Mr}\end{array}$ & $\begin{array}{l}\left({ }^{\circ} \mathrm{C}\right) \\
\text { um }\end{array}$ & \multicolumn{2}{|c|}{$\begin{array}{c}\text { 降雨量 } \\
\text { Rainfall } \\
(\mathrm{mm})\end{array}$} & \multicolumn{2}{|c|}{$\begin{array}{c}\text { 平均湿度 } \\
\text { Average relative } \\
\text { humidity (\%) }\end{array}$} & \multicolumn{2}{|c|}{$\begin{array}{c}\text { 总日照时数 } \\
\text { Total sunshine } \\
\text { hours (h) }\end{array}$} \\
\hline & 2016 & 2017 & 2016 & 2017 & 2016 & 2017 & 2016 & 2017 & 2016 & 2017 \\
\hline 5 月 May & 10.5 & 11.0 & 24.2 & 25.9 & 175.7 & 37.1 & 51.7 & 49.5 & 257.5 & 292.8 \\
\hline 6 月 June & 16.1 & 15.8 & 27.7 & 29.3 & 170.3 & 64.9 & 66.2 & 56.7 & 204.0 & 268.4 \\
\hline 7 月 July & 20.4 & 21.2 & 30.0 & 31.7 & 363.7 & 109.8 & 77.2 & 71.8 & 188.4 & 206.5 \\
\hline 8 月 August & 19.7 & 18.8 & 29.6 & 29.3 & 68.6 & 128.1 & 75.8 & 80.7 & 224.7 & 170.4 \\
\hline 9 月 September & 13.7 & 11.4 & 24.7 & 25.3 & 40.5 & 57.6 & 77.7 & 72.9 & 233.7 & 207.5 \\
\hline
\end{tabular}

表 2 基藮肥与穗肥施用分配(施氮总量 $225 \mathbf{k g ~ h m}^{-2}$ )

Table 2 Ratio and applied amount of base tillering fertilizer and panicle fertilizer (total $\left.\mathbf{N} 225 \mathrm{~kg} \mathrm{hm}^{-2}\right)\left(\mathrm{kg} \mathrm{hm}^{-2}\right)$

\begin{tabular}{|c|c|c|c|c|}
\hline $\begin{array}{c}\text { 基蒒肥:穗肥 } \\
\text { B:P }\end{array}$ & $\begin{array}{c}\text { 基肥 } \\
\text { Basal } \\
\text { fertilizer }\end{array}$ & $\begin{array}{c}\text { 藍肥 } \\
\text { Tillering } \\
\text { fertilizer }\end{array}$ & $\begin{array}{c}\text { 基肥+蔁肥 } \\
\text { Basal fertilizer and } \\
\text { tillering fertilizer }\end{array}$ & $\begin{array}{c}\text { 穗肥 } \\
\text { Panicle } \\
\text { fertilizer }\end{array}$ \\
\hline $8: 2$ & 108 & 72 & 180 & 45 \\
\hline $6: 4$ & 81 & 54 & 135 & 90 \\
\hline
\end{tabular}

$\mathrm{B}: \mathrm{P}$ 为基藍肥:穗肥。 $\mathrm{B}: \mathrm{P}$ means basal tillering fertilizer: panicle fertilizer.

表 3 不同品种萻肥与穗肥叶龄组合

Table 3 Leaf age combination of tillering fertilizer and ear fertilizer in different varieties

\begin{tabular}{|c|c|c|c|c|c|c|c|c|}
\hline \multicolumn{3}{|c|}{ 吉粳 88 Jigeng 88} & \multicolumn{3}{|c|}{ 沈农 265 Shennong 265} & \multicolumn{3}{|c|}{ 沈农 1401 Shennong 1401} \\
\hline $\begin{array}{c}\text { 叶龄 } \\
\text { (叶龄指数) } \\
\text { Leaf ages } \\
\text { (leaf age index) }\end{array}$ & $\begin{array}{c}11 \text { 叶 } \\
11 \text { leaves } \\
(78.6 \%)\end{array}$ & $\begin{array}{c}13 \text { 叶 } \\
13 \text { leaves } \\
(92.9 \%)\end{array}$ & $\begin{array}{c}\text { 叶龄 } \\
\text { (叶龄指数) } \\
\text { Leaf ages } \\
\text { (leaf age index) }\end{array}$ & $\begin{array}{c}12 \text { 叶 } \\
12 \text { leaves } \\
(80.0 \%)\end{array}$ & $\begin{array}{c}14 \text { 叶 } \\
14 \text { leaves } \\
(93.3 \%)\end{array}$ & $\begin{array}{c}\text { 叶龄 } \\
\text { (叶龄指数) } \\
\text { Leaf ages } \\
\text { (leaf age index) }\end{array}$ & $\begin{array}{c}13 \text { 叶 } \\
13 \text { leaves } \\
(81.3 \%)\end{array}$ & $\begin{array}{c}15 \text { 叶 } \\
15 \text { leaves } \\
(93.6 \%)\end{array}$ \\
\hline $6(42.9 \%)$ & $(6,11)^{\#}$ & $(6,13)$ & $7(46.7 \%)$ & $(7,12)$ & $(7,14)$ & $8(50.0 \%)$ & $(8,13)$ & $(8,15)$ \\
\hline $7(50.0 \%)$ & $(7,11)$ & $(7,13)$ & $8(53.3 \%)$ & $(8,12)$ & $(8,14)$ & $9(56.3 \%)$ & $(9,13)$ & $(9,15)$ \\
\hline $8(57.1 \%)$ & $(8,11)$ & $(8,13)$ & $9(60.0 \%)$ & $(9,12)$ & $(9,14)$ & $10(62.5 \%)$ & $(10,13)$ & $(10,15)$ \\
\hline
\end{tabular}

莉肥于 $6 、 7 、 8 、 9$ 叶分别施入，穗肥于 $11 、 12 、 13 、 14 、 15$ 叶分别施入。 ${ }^{*}(6,11)$ 组合表示于第 6 叶完全抽出时施入葟肥，于第 11 叶完全抽出时施入穗肥; 其他依此类推。

Tillering fertilizer was applied when the 6, 7, 8, 9 leaves extracted, panicle fertilizer was applied when the 11, 12, 13, 14, 15 leaves extracted. \# $(6,11)$ means that the tillering fertilizer was applied when the sixth leaf was fully extracted, and the panicle fertilizer was applied when the eleventh leaf was completely extracted; the rest may be deduced by analogy.

\section{3 测定项目}

\subsection{1 叶龄动态移栽后 2 d调查标定叶龄数, 从} 6 月 1 日开始每隔5 d调查叶龄进程，直至主茎叶片全 部抽出。

\subsection{2 茎雀成穗率 从移栽后 $15 \mathrm{~d}$ 开始调查, 每} 隔 $5 \mathrm{~d}$ 普查筒栽全部植株, 并记录每穴茎藍数消长动 态，直至抽穗期结束，计算成穗率。
1.3.3 分化颖花数 在颖花分化期, 选取每筒处 理中第1穴内大小均匀、长势基本一致的 3 个穗，使 用解剖镜分别观察测定每穗总颖花数 ${ }^{[17]}$ 。

1.3.4 实际颖花数在水稻成熟期, 选取每筒处 理中第2穴，对其中大小均匀、长势基本一致的 3 个 穗, 观察测定每穗实粒数和秕粒数。

1.3.5 考种收取筒栽所有稻穗，每穴单独分装 
标记，风干后进行室内考种，包括穗数、穗粒数、结 实率、千粒重等。

\subsection{6 氮素含量考种后, 将所有处理按茎鞘、}

叶、穗分别烘干至恒重, 并用FZ102微型植物粉碎 机磨粉, 从每个处理称取 $0.5 \mathrm{~g}$ 混合样置于玻璃消 解管中, 加 $\mathrm{H}_{2} \mathrm{SO}_{4}{ }^{+}$定氮催化片消煮, 后用丹麦进 口的FOSS全自动凯氏定氮仪Kijeltec 8400 测定氮 素含量。

\section{4 数据计算和统计分析}

实际颖花数 $=$ 结实颖花数 + 空癔颖花数;

退化颖花数 $=$ 分化颖花数 - 实际颖花数;

氮素积累总量 $\left(\mathrm{kg} \mathrm{hm}^{-2}\right)=$ 成熟期单位面积植株 (茎鞘、叶、穗)氮积累量的总和;

氮素吸收利用率 $(\%)=($ 施氮区植株总吸氮量 - 空 白区植株总吸氮量 $) /$ 施氮量 $\times 100$;

氮素农学利用率 $\left(\mathrm{kg} \mathrm{kg}^{-1}\right)=($ 施氮区产量一空白区 产量)/施氮量;

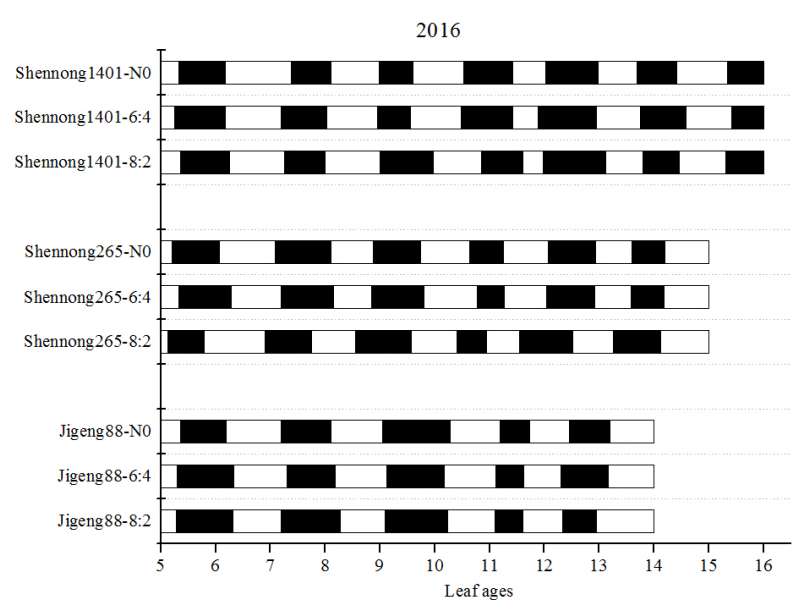

氮素偏生产力 $\left(\mathrm{kg} \mathrm{kg}^{-1}\right)=$ 籽粒产量/施氮量。

运用Microsoft Excel 2010软件录入数据, Origin 9软件制作图形, SPSS19软件进行统计分析。

\section{2 结果与分析}

\section{1 叶龄进程}

由图 1 可知, 从整个生育期叶片生长速度来看, 同一品种不同叶片的出叶速度及持续时间略有差 异, 但差异不明显, 不同氮肥运筹及空白(N0)条件 下均如此。出叶速度最快的叶片均在 8 叶以后, 吉 粳 88 在 8.3 9.2 叶间、沈农 265 在 8.2 10.2 叶间、 沈农 1401 在 8.9 11.7 叶间出叶速度相对最快。在 全生育时期总施氮量一致的条件下，不同品种 2 种 氮肥运筹与相应空白对照 $(\mathrm{N} 0)$ 相比，在叶龄进程、 主茎叶片数上均无显著差异, 说明水稻生育进程及 生育期主要受遗传特性决定, 与外源氮素及其施用 方式无关。

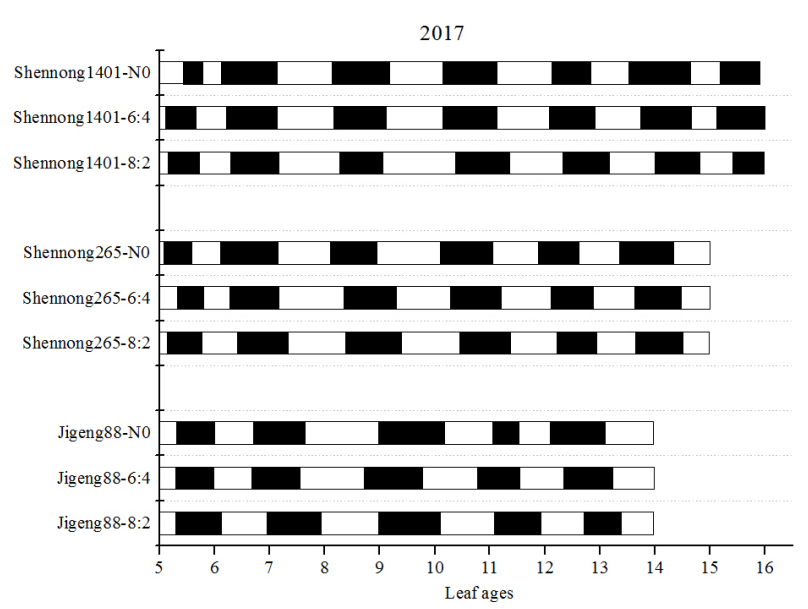

图 1 不同氮肥运筹下各处理每 $5 \mathrm{~d}$ 叶龄进程

Fig. 1 Leaf age process of every five days under different nitrogen applications 图中横坐标始于 5, 表示叶龄为 5 叶时移栽，每条柱中各小段表示每 $5 \mathrm{~d}$ 的叶龄进程。

The abscissa in the picture starts at 5, said leaf transplanting age of 5 leaves. Each section in each bar represents the process of leaf age growth every five days.

\section{2 茎菜成穗率}

在不同氮肥运筹下 3 个水稻品种的茎䔉成穗率 存在显著差异, 且氮肥运筹 $6: 4$ 处理下的成穗率显 著大于8：2处理, 2年规律基本一致。2016年和 2017 年吉粳 88 在氮肥运筹 $8: 2$ 处理下平均成穗率分别为 $64.3 \%$ 和 $63.2 \%$, 在 6: 4 处理下平均成穗率分别为 $78.6 \%$ 和 $74.8 \%$; 其中处理 $6: 4(8,11)$ 的成穗率最大, 两年分别为 $87.3 \%$ 和 $80.3 \%$ 。沈农 265 在氮肥运筹 $8: 2$ 处理下 2 年的平均成穗率分别为 $59.4 \%$ 和 $65.9 \%$, 在
6：4处理下分别为 $75.4 \%$ 和 $73.5 \%$; 其中处理 $6 ： 4(9$, 12 )的平均成穗率达到最大， 2 年分别为 $82.5 \%$ 和 $85.5 \%$ 。沈农 1401 在氮肥运筹 $8 ： 2$ 处理下 2 年的平均 成穗率分别为 $61.6 \%$ 和 $52.0 \%$, 在 $6: 4$ 处理下分别为 $69.8 \%$ 和 $65.1 \%$, 其中处理 $6: 4(10,13)$ 的平均成穗 率达到最大, 分别为 $86.4 \%$ 和 $81.2 \%$ 。氮肥施用比例 后移、蒒菜肥施用时期后移均显著提高了成穗率，起 到了保藍效果。在同一氮肥运筹下, 2 年 3 个品种的茎 菜成穗率均随藮肥施用叶龄的延后而增加(图2)。 

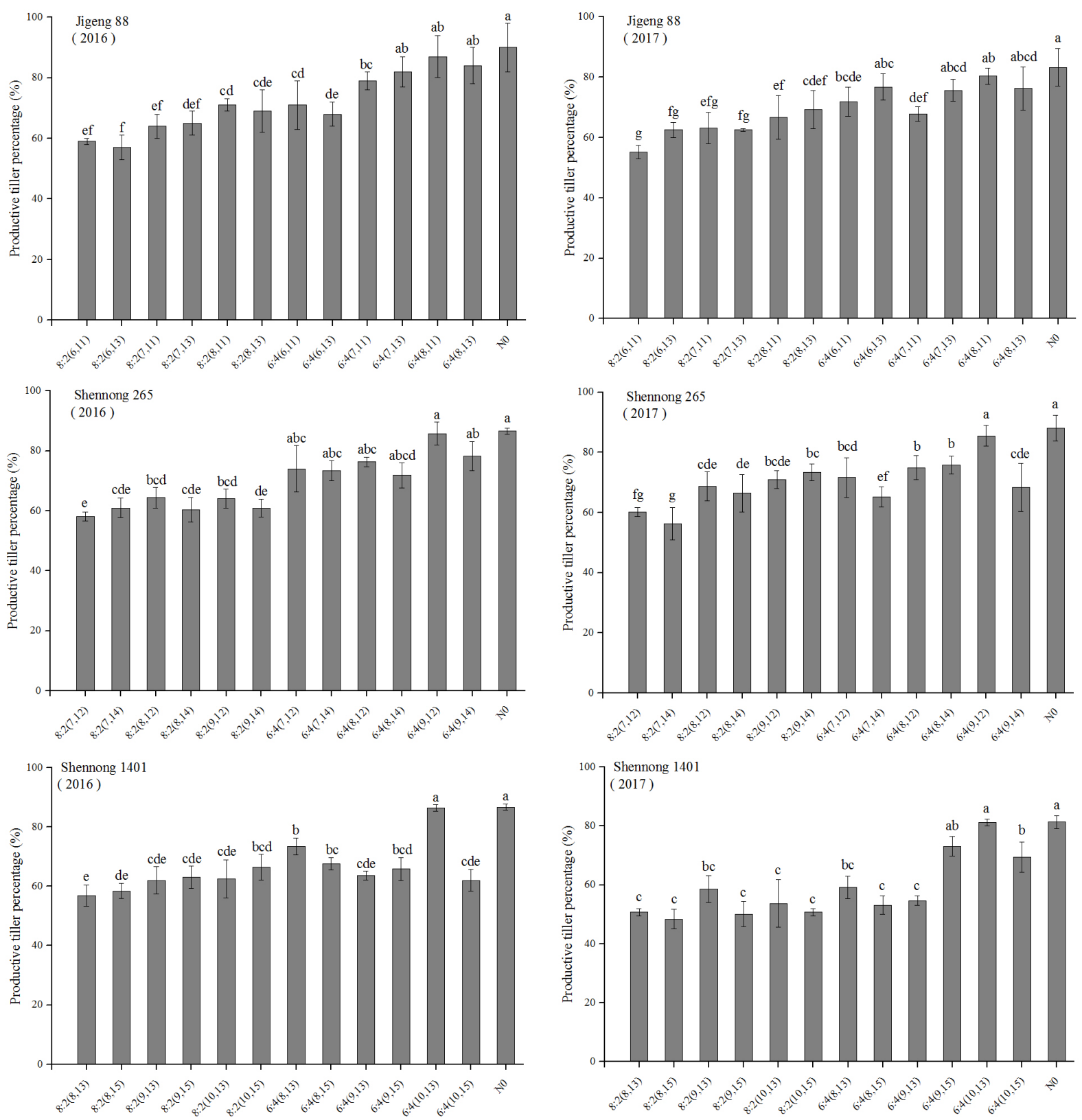

图 2 不同氮肥运筹下各处理茎藮成穗率(2016-2017)

Fig. 2 Productive tiller percentage of rice for different treatments under different nitrogen applications (2016-2017) 图中所标注的字母为成穗率的显著性。不同字母表示在 0.05 水平上差异显著。

Bars superscripted by different letters are significantly different at $P<0.05$.

\section{3 群体颖花数}

由图 3 可知, 2 种氮肥运筹下不同品种的最大分 化颖花数的菜、穗肥叶龄组合分别是 8 叶和 11 叶(吉 粳 88)、9 叶和 12 叶(沈农 265)、10 叶和 13 叶(沈农 1401), 且氮肥运筹 6：4 总体均高于氮肥运筹 8：2。 在同一施用穗肥的叶龄下, 3 个不同生育期水稻品种 的最大分化颖花数和实际颖花数均随葟肥施用的延 后而增加; 在同一施用䆺肥的叶龄下, 3 个水稻品种
的最大分化颖花数和实际颖花数整体上均表现为 倒 4 叶穗肥处理下高于倒 2 叶处理, 且倒 4 叶穗肥 处理下 3 个水稻品种的退化颖花数明显高于倒 2 叶 处理, 但空癔颖花数差异不明显, 2 年试验结果趋 势基本一致。说明施氮量和薛肥施用时期适度后 移、穗肥适当前移，有利于提高分化䓉花数和实际 颖花数。2016 年水稻植株的空痻颖花数整体上较 2017 年高。 

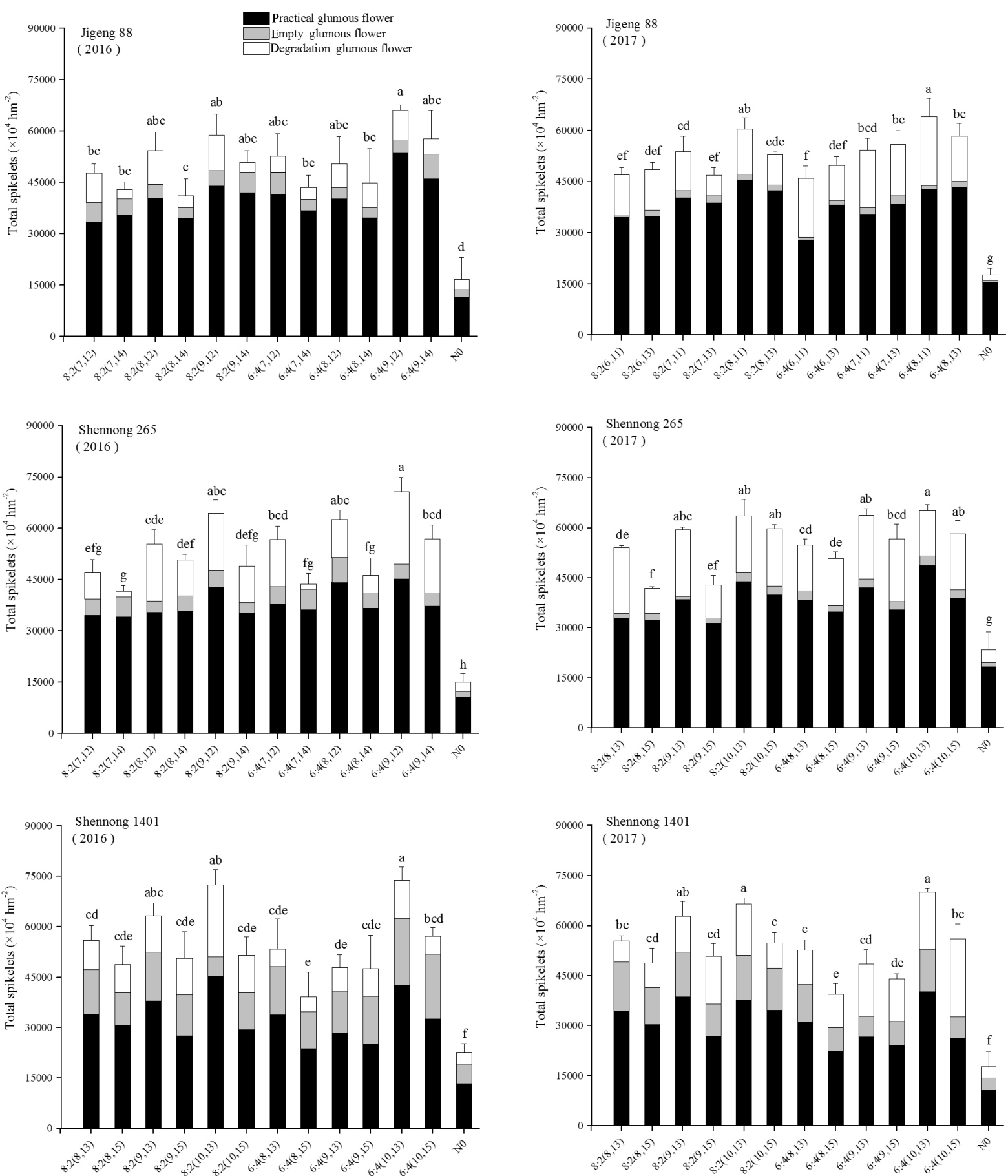

图 3 氮肥运筹对不同品种粳稻颖花量的影响(2016-2017)

Fig. 3 Effect of nitrogen application on the flower volume of japonica rice in cultivars (2016-2017)

图中所标注的字母为分化颖花数的显著性, 即实际颖花数、空癔颖花数与退化颖花数之和。不同字母表示在 0.05 水平上差异显著。 The letters marked in the figure are significant for the number of spikelets, which is the sum of the number of degradation glumous flower, empty glumous flower and practical glumous flower. Different letters above bars stand for significant differences at $P<0.05$.

\section{4 产量及其构成因素}

由表 4 和表 5 可以看出, 6:4运筹下的不同品种 产量及其构成因素大都显著高于 $8: 2$ 运筹, 2 年呈 相同趋势。在同一比例的氮肥运筹下, 吉粳 88 的 $(8$, $11)$ 、沈农 265 的 $(9,12)$ 和沈农 1401 的 $(10,13)$ 处理的
产量均高于同品种的其他处理，且在6：4运筹下最 高; 在同一穗肥施用叶龄下, 3 个水稻品种的产量 均表现为随藍肥施用叶龄的延后而增加，且总体表 现出产量高者有效穗数也较高。不同穗肥施用叶龄 产量吉粳 88 为 11 叶 $>13$ 叶, 沈农 265 为 12 叶 $>14$ 叶, 
沈农 1401 为 13 叶> 15 叶, 即各处理产量表现趋势为 倒4叶 $>$ 倒2叶, 且总体表现出产量高者穗粒数也较 高。说明适当后移藍肥施用叶龄时期保菜效果最
佳，成穗率显著提高; 穗肥施用叶龄时期前移， 同时提高穗肥施用比例, 可显著促进颖花分化, 增加穗粒数。

表 42016 年不同氮肥运筹对各处理产量及其构成的影响

Table 4 Effects of different nitrogen fertilizers on yield and its component of each variety in 2016

\begin{tabular}{|c|c|c|c|c|c|c|c|}
\hline $\begin{array}{c}\text { 品种 } \\
\text { Cultivar }\end{array}$ & $\begin{array}{c}\text { 基㹂肥:穗肥 } \\
\text { B:P }\end{array}$ & $\begin{array}{l}\text { 处理时期 } \\
\text { Treatment }\end{array}$ & $\begin{array}{c}\text { 每筒有效穗数 } \\
\text { No. of panicles } \\
\text { per pot }\end{array}$ & $\begin{array}{c}\text { 每穗粒数 } \\
\text { No. of grains } \\
\text { per panicle }\end{array}$ & $\begin{array}{c}\text { 结实率 } \\
\text { Filled-grain } \\
\text { percentage (\%) }\end{array}$ & $\begin{array}{c}\text { 千粒重 } \\
\text { 1000-grain } \\
\text { weight (g) }\end{array}$ & $\begin{array}{c}\text { 每筒实 } \\
\text { 测产量 } \\
\text { Yield per pot (g) }\end{array}$ \\
\hline \multirow{13}{*}{$\begin{array}{c}\text { 吉粳 } 88 \\
\text { Jigeng } 88\end{array}$} & \multirow[t]{6}{*}{$8: 2$} & $(6,11)^{\#}$ & $21.6 \pm 2.4 \mathrm{ab}$ & $139.8 \pm 5.9 \mathrm{c}$ & $89.2 \pm 4.0 \mathrm{abcd}$ & $21.3 \pm 0.4 \mathrm{bc}$ & $56.8 \pm 0.8 \mathrm{~b}$ \\
\hline & & $(6,13)$ & $18.2 \pm 0.5 \mathrm{~b}$ & $137.3 \pm 4.7 \mathrm{c}$ & $86.9 \pm 3.5 \mathrm{~cd}$ & $20.4 \pm 0.2 \mathrm{~cd}$ & $52.8 \pm 0.4 \mathrm{~b}$ \\
\hline & & $(7,11)$ & $23.6 \pm 2.6 \mathrm{ab}$ & $139.0 \pm 10.8 \mathrm{c}$ & $88.5 \pm 5.1 \mathrm{abcd}$ & $21.1 \pm 1.2 \mathrm{bc}$ & $60.0 \pm 2.8 \mathrm{~b}$ \\
\hline & & $(7,13)$ & $24.0 \pm 2.1 \mathrm{ab}$ & $120.0 \pm 1.0 \mathrm{~d}$ & $87.1 \pm 0.0 \mathrm{bcd}$ & $21.0 \pm 0.6 \mathrm{bc}$ & $52.8 \pm 3.4 \mathrm{~b}$ \\
\hline & & $(8,11)$ & $26.2 \pm 3.6 \mathrm{a}$ & $146.6 \pm 14.4 \mathrm{abc}$ & $90.5 \pm 0.3 \mathrm{abcd}$ & $19.3 \pm 2.0 \mathrm{~d}$ & $67.2 \pm 5.4 \mathrm{ab}$ \\
\hline & & $(8,13)$ & $23.2 \pm 0.8 \mathrm{~b}$ & $134.8 \pm 4.9 \mathrm{c}$ & $92.1 \pm 0.2 \mathrm{ab}$ & $21.1 \pm 1.1 \mathrm{bc}$ & $60.8 \pm 4.0 \mathrm{~b}$ \\
\hline & \multirow[t]{6}{*}{$6: 4$} & $(6,11)$ & $22.0 \pm 2.0 \mathrm{ab}$ & $159.3 \pm 7.4 \mathrm{a}$ & $86.1 \pm 2.3 \mathrm{~d}$ & $21.0 \pm 0.4 \mathrm{bc}$ & $62.4 \pm 0.4 \mathrm{ab}$ \\
\hline & & $(6,13)$ & $18.2 \pm 0.5 \mathrm{~b}$ & $146.7 \pm 2.1 \mathrm{abc}$ & $91.5 \pm 1.1 \mathrm{abc}$ & $20.8 \pm 0.1 \mathrm{bc}$ & $54.4 \pm 0.4 \mathrm{~b}$ \\
\hline & & $(7,11)$ & $24.6 \pm 1.2 \mathrm{ab}$ & $143.4 \pm 10.5 \mathrm{bc}$ & $91.5 \pm 3.3 \mathrm{~d}$ & $21.1 \pm 1.3 \mathrm{bc}$ & $64.8 \pm 4.0 \mathrm{~b}$ \\
\hline & & $(7,13)$ & $19.8 \pm 1.2 \mathrm{ab}$ & $134.0 \pm 7.9 \mathrm{c}$ & $92.1 \pm 1.4 \mathrm{abcd}$ & $21.9 \pm 0.2 \mathrm{~b}$ & $52.8 \pm 2.8 \mathrm{~b}$ \\
\hline & & $(8,11)$ & $25.2 \pm 1.2 \mathrm{a}$ & $155.4 \pm 11.7 \mathrm{ab}$ & $93.1 \pm 2.2 \mathrm{a}$ & $20.9 \pm 0.6 \mathrm{bc}$ & $76.0 \pm 1.6 \mathrm{a}$ \\
\hline & & $(8,13)$ & $25.0 \pm 1.5 \mathrm{a}$ & $136.4 \pm 11.3 \mathrm{c}$ & $91.1 \pm 2.5 \mathrm{abc}$ & $20.5 \pm 0.5 \mathrm{bcd}$ & $63.2 \pm 1.2 \mathrm{ab}$ \\
\hline & No & $(0,0)$ & $10.2 \pm 0.5 \mathrm{c}$ & $114.0 \pm 3.5 \mathrm{~d}$ & $89.5 \pm 2.9 \mathrm{abcd}$ & $23.7 \pm 0.9 \mathrm{a}$ & $24.8 \pm 2.4 \mathrm{c}$ \\
\hline \multirow{13}{*}{$\begin{array}{c}\text { 沈农 } 265 \\
\text { Shennong } \\
265\end{array}$} & \multirow[t]{6}{*}{$8: 2$} & $(7,12)$ & $18.0 \pm 0.8 \mathrm{c}$ & $155.8 \pm 8.3 \mathrm{ab}$ & $86.0 \pm 1.1 \mathrm{def}$ & $24.1 \pm 0.4 \mathrm{abc}$ & $57.6 \pm 1.2 \mathrm{ef}$ \\
\hline & & $(7,14)$ & $18.8 \pm 0.4 \mathrm{c}$ & $147.0 \pm 2.2 \mathrm{bcd}$ & $83.5 \pm 1.4 \mathrm{f}$ & $24.0 \pm 0.2 \mathrm{abc}$ & $55.2 \pm 0.4 \mathrm{f}$ \\
\hline & & $(8,12)$ & $21.2 \pm 1.0 \mathrm{abc}$ & $134.0 \pm 4.5 \mathrm{e}$ & $90.8 \pm 1.0 \mathrm{ab}$ & $23.7 \pm 0.1 \mathrm{bcd}$ & $60.8 \pm 1.6 \mathrm{def}$ \\
\hline & & $(8,14)$ & $21.6 \pm 0.8 \mathrm{abc}$ & $136.8 \pm 7.8 \mathrm{de}$ & $87.8 \pm 2.3 \mathrm{cde}$ & $23.0 \pm 0.6 \mathrm{ef}$ & $59.2 \pm 2.4 \mathrm{ef}$ \\
\hline & & $(9,12)$ & $23.6 \pm 1.4 \mathrm{ab}$ & $149.3 \pm 9.4 \mathrm{bc}$ & $88.8 \pm 1.6 \mathrm{bcd}$ & $22.8 \pm 0.4 \mathrm{f}$ & $71.2 \pm 1.2 \mathrm{~b}$ \\
\hline & & $(9,14)$ & $19.2 \pm 0.7 \mathrm{c}$ & $149.3 \pm 7.3 \mathrm{bc}$ & $91.4 \pm 0.4 \mathrm{a}$ & $23.5 \pm 0.4 \mathrm{de}$ & $61.6 \pm 1.2 \mathrm{def}$ \\
\hline & \multirow[t]{6}{*}{$6: 4$} & $(7,12)$ & $22.0 \pm 2.0 \mathrm{abc}$ & $142.5 \pm 8.2 \mathrm{cde}$ & $86.8 \pm 1.0 \mathrm{cde}$ & $22.8 \pm 0.4 \mathrm{f}$ & $61.6 \pm 3.2 \mathrm{de}$ \\
\hline & & $(7,14)$ & $18.0 \pm 0.8 \mathrm{c}$ & $162.0 \pm 7.8 \mathrm{a}$ & $84.1 \pm 1.8 \mathrm{f}$ & $24.2 \pm 0.1 \mathrm{ab}$ & $59.2 \pm 1.6 \mathrm{ef}$ \\
\hline & & $(8,12)$ & $23.6 \pm 2.0 \mathrm{ab}$ & $155.3 \pm 10.2 \mathrm{ab}$ & $85.2 \pm 3.4 \mathrm{ef}$ & $22.0 \pm 0.3 \mathrm{~g}$ & $68.0 \pm 2.4 \mathrm{bc}$ \\
\hline & & $(8,14)$ & $19.6 \pm 1.4 \mathrm{c}$ & $152.5 \pm 9.9 \mathrm{abc}$ & $88.8 \pm 0.5 \mathrm{bcd}$ & $24.2 \pm 0.2 \mathrm{ab}$ & $64.0 \pm 2.4 \mathrm{cde}$ \\
\hline & & $(9,12)$ & $24.4 \pm 1.4 \mathrm{a}$ & $149.3 \pm 4.5 \mathrm{bc}$ & $91.3 \pm 2.5 \mathrm{ab}$ & $23.2 \pm 0.8 \mathrm{def}$ & $76.8 \pm 1.2 \mathrm{a}$ \\
\hline & & $(9,14)$ & $20.4 \pm 1.2 \mathrm{bc}$ & $150.8 \pm 7.5 \mathrm{abc}$ & $89.7 \pm 3.0 \mathrm{abc}$ & $24.3 \pm 0.1 \mathrm{a}$ & $66.4 \pm 2.4 \mathrm{bcd}$ \\
\hline & No & $(0,0)$ & $10.4 \pm 0.7 \mathrm{~d}$ & $115.0 \pm 4.2 \mathrm{f}$ & $95.4 \pm 0.7 \mathrm{a}$ & $24.2 \pm 0.2 \mathrm{ab}$ & $27.2 \pm 1.2 \mathrm{~g}$ \\
\hline \multirow{6}{*}{$\begin{array}{c}\text { 沈农 } 1401 \\
\text { Shennong } \\
1401\end{array}$} & \multirow[t]{6}{*}{$8: 2$} & $(8,13)$ & $18.0 \pm 0.8 \mathrm{abcd}$ & $142.3 \pm 18.2 \mathrm{abc}$ & $70.3 \pm 9.0 \mathrm{abcd}$ & $26.0 \pm 1.0 \mathrm{ab}$ & $66.4 \pm 4.4 \mathrm{bcd}$ \\
\hline & & $(8,15)$ & $15.6 \pm 1.4 \mathrm{de}$ & $144.5 \pm 4.0 \mathrm{abc}$ & $76.8 \pm 1.3 \mathrm{a}$ & $26.5 \pm 0.7 \mathrm{a}$ & $59.2 \pm 3.6 \mathrm{de}$ \\
\hline & & $(9,13)$ & $17.6 \pm 1.1 \mathrm{bcde}$ & $133.3 \pm 8.4 \mathrm{~cd}$ & $66.3 \pm 8.7 \mathrm{abcd}$ & $25.2 \pm 0.5 \mathrm{abc}$ & $59.2 \pm 2.8 \mathrm{de}$ \\
\hline & & $(9,15)$ & $17.2 \pm 0.4 \mathrm{bcde}$ & $119.8 \pm 9.3 \mathrm{~d}$ & $62.4 \pm 10.9 \mathrm{bcd}$ & $26.7 \pm 0.8 \mathrm{a}$ & $55.2 \pm 2.4 \mathrm{e}$ \\
\hline & & $(10,13)$ & $18.4 \pm 0.5 \mathrm{abc}$ & $152.3 \pm 12.6 \mathrm{ab}$ & $64.1 \pm 6.3 \mathrm{abcd}$ & $24.7 \pm 0.8 \mathrm{bc}$ & $68.8 \pm 3.6 \mathrm{abc}$ \\
\hline & & $(10,15)$ & $16.0 \pm 0.7 \mathrm{cde}$ & $138.5 \pm 15.9 \mathrm{bc}$ & $70.6 \pm 10.3 \mathrm{abc}$ & $26.2 \pm 1.1 \mathrm{ab}$ & $57.6 \pm 3.2 \mathrm{de}$ \\
\hline
\end{tabular}


(续表 4)

\begin{tabular}{|c|c|c|c|c|c|c|c|}
\hline $\begin{array}{c}\text { 品种 } \\
\text { Cultivar }\end{array}$ & $\begin{array}{c}\text { 基菜肥:穗肥 } \\
\text { B:P }\end{array}$ & $\begin{array}{l}\text { 处理时期 } \\
\text { Treatment }\end{array}$ & $\begin{array}{c}\text { 每筒有效穗数 } \\
\text { No. of panicles } \\
\text { per pot }\end{array}$ & $\begin{array}{c}\text { 每穗粒数 } \\
\text { No. of grains } \\
\text { per panicle }\end{array}$ & $\begin{array}{c}\text { 结实率 } \\
\text { Filled-grain } \\
\text { percentage (\%) }\end{array}$ & $\begin{array}{c}\text { 千粒重 } \\
\text { 1000-grain } \\
\text { weight (g) }\end{array}$ & $\begin{array}{c}\text { 每筒实 } \\
\text { 测产量 } \\
\text { Yield per pot }(\mathrm{g})\end{array}$ \\
\hline \multirow{7}{*}{$\begin{array}{c}\text { 沈农 } 1401 \\
\text { Shennong } \\
1401\end{array}$} & $6: 4$ & $(8,13)$ & $18.8 \pm 1.0 \mathrm{ab}$ & $151.5 \pm 5.4 \mathrm{ab}$ & $61.2 \pm 3.9 \mathrm{~cd}$ & $25.4 \pm 0.3 \mathrm{abc}$ & $72.0 \pm 2.4 \mathrm{ab}$ \\
\hline & & $(8,15)$ & $17.2 \pm 0.4$ bcde & $138.5 \pm 1.7 \mathrm{bc}$ & $63.6 \pm 4.0 \mathrm{abcd}$ & $26.1 \pm 0.9 \mathrm{ab}$ & $62.4 \pm 0.8 \mathrm{cde}$ \\
\hline & & $(9,13)$ & $18.4 \pm 0.5 \mathrm{abc}$ & $131.3 \pm 4.3 \mathrm{~cd}$ & $56.7 \pm 5.8 \mathrm{~d}$ & $26.6 \pm 0.6 \mathrm{a}$ & $64.0 \pm 1.2 \mathrm{bcd}$ \\
\hline & & $(9,15)$ & $16.0 \pm 0.7 \mathrm{cde}$ & $144.8 \pm 4.5 \mathrm{abc}$ & $75.3 \pm 5.3 \mathrm{ab}$ & $26.0 \pm 0.6 \mathrm{ab}$ & $60.0 \pm 2.0 \mathrm{de}$ \\
\hline & & $(10,13)$ & $20.4 \pm 1.0 \mathrm{a}$ & $155.3 \pm 8.1 \mathrm{a}$ & $71.3 \pm 6.7 \mathrm{abc}$ & $24.1 \pm 1.9 \mathrm{c}$ & $76.0 \pm 1.6 \mathrm{a}$ \\
\hline & & $(10,15)$ & $15.2 \pm 0.5 \mathrm{e}$ & $149.3 \pm 11.2 \mathrm{ab}$ & $66.5 \pm 7.6 \mathrm{abcd}$ & $26.2 \pm 1.3 \mathrm{ab}$ & $59.2 \pm 1.6 \mathrm{de}$ \\
\hline & No & $(0,0)$ & $8.0 \pm 0.7 \mathrm{f}$ & $103.0 \pm 4.5 \mathrm{e}$ & $64.9 \pm 9.3 \mathrm{abcd}$ & $25.5 \pm 0.8 \mathrm{abc}$ & $20.8 \pm 1.2 \mathrm{f}$ \\
\hline
\end{tabular}

葲肥于 $6 、 7 、 8 、 9$ 叶分别施入, 穗肥于 $11 、 12 、 13 、 14 、 15$ 叶分别施入。 ${ }^{*}(6,11)$ 组合表示于第 6 叶完全抽出时施入䵒肥, 于第 11 叶完全抽出时施入穗肥; 其他依此类推。 $\mathrm{B}: \mathrm{P}$ 为基藮肥：穗肥。相同品种，同一列中不同小写字母表示在 0.05 水平上差异显著。

Tillering fertilizer was applied when the 6, 7, 8, 9 leaves extracted, panicle fertilizer was applied when the 11,12,13,14, 15 leaves extracted. \# $(6,11)$ means that the tillering fertilizer was applied when the sixth leaf was fully extracted, and the panicle fertilizer was applied when the eleventh leaf was completely extracted; the rest may be deduced by analogy. B:P means basal tillering fertilizer : panicle fertilizer. Values within a column followed by different letters are significantly different at $P<0.05$ in the same cultivar.

\section{表 52017 年不同氮肥运筹对各处理产量及其构成的影响}

Table 5 Effects of different nitrogen fertilizers on yield and its component of each variety in 2017

\begin{tabular}{|c|c|c|c|c|c|c|c|}
\hline $\begin{array}{c}\text { 品种 } \\
\text { Cultivar }\end{array}$ & $\begin{array}{c}\text { 基㒘肥:穗肥 } \\
\text { B:P }\end{array}$ & $\begin{array}{l}\text { 处理时期 } \\
\text { Treatment }\end{array}$ & $\begin{array}{c}\text { 每筒有效穗数 } \\
\text { No. of panicles } \\
\text { per pot }\end{array}$ & $\begin{array}{c}\text { 每穗粒数 } \\
\text { No. of grains } \\
\text { per panicle }\end{array}$ & $\begin{array}{c}\text { 结实率 } \\
\text { Filled-grain } \\
\text { percentage }(\%)\end{array}$ & $\begin{array}{c}\text { 千粒重 } \\
\text { 1000-grain } \\
\text { weight (g) }\end{array}$ & $\begin{array}{c}\text { 每筒实测产量 } \\
\text { Yield per } \\
\text { pot }(\mathrm{g})\end{array}$ \\
\hline \multirow{13}{*}{$\begin{array}{c}\text { 吉粳 } 88 \\
\text { Jigeng } 88\end{array}$} & \multirow[t]{6}{*}{$8: 2$} & $(6,11)^{\#}$ & $19.2 \pm 1.6 \mathrm{c}$ & $146.7 \pm 5.7 \mathrm{bc}$ & $97.4 \pm 1.2 \mathrm{a}$ & $24.2 \pm 1.3 \mathrm{ab}$ & $66.4 \pm 5.2 \mathrm{abc}$ \\
\hline & & $(6,13)$ & $19.4 \pm 0.6 \mathrm{c}$ & $137.0 \pm 5.2 \mathrm{~d}$ & $96.5 \pm 2.8 \mathrm{a}$ & $22.8 \pm 1.3 \mathrm{bc}$ & $58.4 \pm 2.4 \mathrm{bc}$ \\
\hline & & $(7,11)$ & $21.4 \pm 0.4 \mathrm{bc}$ & $159.0 \pm 19.3 \mathrm{bc}$ & $96.0 \pm 1.2 \mathrm{a}$ & $22.5 \pm 0.7 \mathrm{bc}$ & $72.8 \pm 3.2 \mathrm{abc}$ \\
\hline & & $(7,13)$ & $19.2 \pm 1.3 \mathrm{c}$ & $153.3 \pm 22.2 \mathrm{bc}$ & $94.2 \pm 4.1 \mathrm{a}$ & $22.4 \pm 0.5 \mathrm{bc}$ & $60.8 \pm 3.2 \mathrm{abc}$ \\
\hline & & $(8,11)$ & $22.4 \pm 0.7 \mathrm{ab}$ & $170.7 \pm 7.4 \mathrm{ab}$ & $96.5 \pm 0.9 \mathrm{a}$ & $22.6 \pm 0.9 \mathrm{bc}$ & $84.0 \pm 5.6 \mathrm{a}$ \\
\hline & & $(8,13)$ & $21.8 \pm 0.8 \mathrm{~b}$ & $166.0 \pm 28.4 \mathrm{ab}$ & $94.4 \pm 2.7 \mathrm{a}$ & $21.6 \pm 0.6 \mathrm{c}$ & $74.4 \pm 3.8 \mathrm{abc}$ \\
\hline & \multirow[t]{6}{*}{$6: 4$} & $(6,11)$ & $20.8 \pm 0.8 \mathrm{bc}$ & $141.7 \pm 3.1 \mathrm{bc}$ & $98.4 \pm 0.7 \mathrm{a}$ & $23.1 \pm 0.4 \mathrm{bc}$ & $67.2 \pm 3.6 \mathrm{abc}$ \\
\hline & & $(6,13)$ & $17.4 \pm 0.6 \mathrm{c}$ & $137.3 \pm 7.5 \mathrm{~cd}$ & $94.9 \pm 4.1 \mathrm{a}$ & $26.5 \pm 2.4 \mathrm{a}$ & $59.2 \pm 1.2 \mathrm{abc}$ \\
\hline & & $(7,11)$ & $19.2 \pm 1.6 \mathrm{c}$ & $146.7 \pm 5.7 \mathrm{bc}$ & $97.4 \pm 1.2 \mathrm{a}$ & $24.2 \pm 1.3 \mathrm{ab}$ & $66.4 \pm 5.2 \mathrm{abc}$ \\
\hline & & $(7,13)$ & $19.2 \pm 1.3 \mathrm{c}$ & $164.0 \pm 9.2 \mathrm{ab}$ & $95.4 \pm 2.8 \mathrm{a}$ & $23.4 \pm 0.6 \mathrm{abc}$ & $70.4 \pm 4.8 \mathrm{abc}$ \\
\hline & & $(8,11)$ & $24.0 \pm 1.1 \mathrm{ab}$ & $175.7 \pm 39.7 \mathrm{ab}$ & $95.9 \pm 1.5 \mathrm{a}$ & $21.5 \pm 0.3 \mathrm{c}$ & $84.8 \pm 6.4 \mathrm{a}$ \\
\hline & & $(8,13)$ & $21.8 \pm 0.8 \mathrm{~b}$ & $161.3 \pm 15.4 \mathrm{bc}$ & $96.8 \pm 1.1 \mathrm{a}$ & $21.6 \pm 0.6 \mathrm{c}$ & $72.8 \pm 1.2 \mathrm{abc}$ \\
\hline & No & $(0,0)$ & $8.6 \pm 0.4 \mathrm{~d}$ & $137.3 \pm 17.3 \mathrm{~cd}$ & $98.6 \pm 1.6 \mathrm{a}$ & $23.3 \pm 0.2 \mathrm{abc}$ & $26.4 \pm 1.2 \mathrm{~d}$ \\
\hline \multirow{8}{*}{$\begin{array}{c}\text { 沈农 } 265 \\
\text { Shennong } \\
265\end{array}$} & \multirow[t]{6}{*}{$8: 2$} & $(7,12)$ & $19.2 \pm 2.1 \mathrm{abcd}$ & $152.5 \pm 33.4 \mathrm{ab}$ & $95.2 \pm 0.8 \mathrm{ab}$ & $25.1 \pm 1.1 \mathrm{ab}$ & $72.8 \pm 8.0 \mathrm{bc}$ \\
\hline & & $(7,14)$ & $17.6 \pm 0.7 \mathrm{bcd}$ & $147.2 \pm 23.0 \mathrm{bc}$ & $94.1 \pm 1.3 \mathrm{~b}$ & $24.8 \pm 0.8 \mathrm{abc}$ & $60.8 \pm 3.6 \mathrm{~d}$ \\
\hline & & $(8,12)$ & $21.8 \pm 1.1 \mathrm{abc}$ & $140.8 \pm 10.7 \mathrm{bc}$ & $97.4 \pm 0.9 \mathrm{a}$ & $24.7 \pm 0.5 \mathrm{abc}$ & $74.4 \pm 5.6 \mathrm{bc}$ \\
\hline & & $(8,14)$ & $17.0 \pm 1.7 \mathrm{~cd}$ & $147.7 \pm 6.7 \mathrm{bc}$ & $95.2 \pm 1.2 \mathrm{ab}$ & $25.6 \pm 0.7 \mathrm{a}$ & $61.6 \pm 3.4 \mathrm{~d}$ \\
\hline & & $(9,12)$ & $23.4 \pm 1.7 \mathrm{a}$ & $146.6 \pm 10.9 \mathrm{bc}$ & $93.3 \pm 2.3 \mathrm{~b}$ & $24.3 \pm 0.6 \mathrm{bc}$ & $79.2 \pm 4.6 \mathrm{ab}$ \\
\hline & & $(9,14)$ & $23.0 \pm 2.1 \mathrm{ab}$ & $133.2 \pm 7.5 \mathrm{~cd}$ & $94.1 \pm 2.4 \mathrm{~b}$ & $23.7 \pm 0.9 \mathrm{c}$ & $68.0 \pm 3.4 \mathrm{~cd}$ \\
\hline & \multirow[t]{2}{*}{$6: 4$} & $(7,12)$ & $21.8 \pm 1.1 \mathrm{abc}$ & $156.5 \pm 23.8 \mathrm{ab}$ & $93.7 \pm 1.3 \mathrm{~b}$ & $23.7 \pm 1.0 \mathrm{c}$ & $76.8 \pm 4.2 \mathrm{~b}$ \\
\hline & & $(7,14)$ & $19.8 \pm 1.1 \mathrm{abcd}$ & $151.0 \pm 21.5 \mathrm{ab}$ & $94.6 \pm 1.3 \mathrm{~b}$ & $24.9 \pm 0.5 \mathrm{abc}$ & $71.2 \pm 4.2 \mathrm{~cd}$ \\
\hline
\end{tabular}


(续表 5)

\begin{tabular}{|c|c|c|c|c|c|c|c|}
\hline $\begin{array}{c}\text { 品种 } \\
\text { Cultivar }\end{array}$ & $\begin{array}{c}\text { 基葟肥:穗肥 } \\
\text { B:P }\end{array}$ & $\begin{array}{l}\text { 处理时期 } \\
\text { Treatment }\end{array}$ & $\begin{array}{c}\text { 每筒有效穗数 } \\
\text { No. of panicles } \\
\text { per pot }\end{array}$ & $\begin{array}{c}\text { 每穗粒数 } \\
\text { No. of grains } \\
\text { per panicle }\end{array}$ & $\begin{array}{c}\text { 结实率 } \\
\text { Filled-grain } \\
\text { percentage (\%) }\end{array}$ & $\begin{array}{c}\text { 千粒重 } \\
\text { 1000-grain } \\
\text { weight (g) }\end{array}$ & $\begin{array}{c}\text { 每筒实测产量 } \\
\text { Yield per } \\
\text { pot }(\mathrm{g})\end{array}$ \\
\hline \multirow{5}{*}{$\begin{array}{c}\text { 沈农 } 265 \\
\text { Shennong } \\
265\end{array}$} & & $(8,12)$ & $20.2 \pm 0.9 \mathrm{abcd}$ & $160.7 \pm 3.8 \mathrm{a}$ & $94.8 \pm 2.4 \mathrm{~b}$ & $24.9 \pm 1.1 \mathrm{abc}$ & $76.8 \pm 3.6 \mathrm{~b}$ \\
\hline & & $(8,14)$ & $23.0 \pm 0.4 \mathrm{ab}$ & $146.8 \pm 24.3 \mathrm{bc}$ & $93.9 \pm 3.0 \mathrm{~b}$ & $22.4 \pm 1.0 \mathrm{~d}$ & $71.2 \pm 4.0 \mathrm{~cd}$ \\
\hline & & $(9,12)$ & $23.4 \pm 2.2 \mathrm{a}$ & $163.7 \pm 11.7 \mathrm{a}$ & $93.0 \pm 1.9 \mathrm{~b}$ & $22.5 \pm 1.4 \mathrm{~d}$ & $81.6 \pm 5.8 \mathrm{a}$ \\
\hline & & $(9,14)$ & $19.8 \pm 2.3 \mathrm{abcd}$ & $149.7 \pm 14.0 \mathrm{bc}$ & $94.8 \pm 1.9 \mathrm{~b}$ & $24.3 \pm 0.7 \mathrm{bc}$ & $68.8 \pm 5.4 \mathrm{~cd}$ \\
\hline & No & $(0,0)$ & $12.6 \pm 1.7 \mathrm{~d}$ & $117.5 \pm 11.9 \mathrm{~d}$ & $93.9 \pm 2.1 \mathrm{~b}$ & $24.5 \pm 0.7 \mathrm{abc}$ & $34.4 \pm 2.8 \mathrm{e}$ \\
\hline \multirow{13}{*}{$\begin{array}{c}\text { 沈农 } 1401 \\
\text { Shennong } \\
1401\end{array}$} & $8: 2$ & $(8,13)$ & $17.0 \pm 0.1 \mathrm{bcd}$ & $155.5 \pm 12.1 \mathrm{ab}$ & $65.5 \pm 1.7 \mathrm{ab}$ & $25.0 \pm 1.0 \mathrm{bc}$ & $56.0 \pm 2.4 \mathrm{bc}$ \\
\hline & & $(8,15)$ & $13.4 \pm 0.5 \mathrm{~d}$ & $148.6 \pm 10.1 \mathrm{abc}$ & $66.9 \pm 2.6 \mathrm{ab}$ & $25.6 \pm 1.0 \mathrm{ab}$ & $48.0 \pm 4.0 \mathrm{de}$ \\
\hline & & $(9,13)$ & $15.0 \pm 0.6 \mathrm{~cd}$ & $157.5 \pm 11.7 \mathrm{ab}$ & $74.6 \pm 4.9 \mathrm{ab}$ & $27.0 \pm 0.9 \mathrm{a}$ & $61.6 \pm 4.2 \mathrm{~b}$ \\
\hline & & $(9,15)$ & $14.4 \pm 0.4 \mathrm{~cd}$ & $124.2 \pm 11.8 \mathrm{bc}$ & $65.0 \pm 3.3 \mathrm{ab}$ & $26.8 \pm 1.6 \mathrm{a}$ & $41.6 \pm 3.6 \mathrm{e}$ \\
\hline & & $(10,13)$ & $20.8 \pm 0.8 \mathrm{ab}$ & $156.5 \pm 9.5 \mathrm{ab}$ & $80.5 \pm 5.6 \mathrm{a}$ & $24.3 \pm 0.8 \mathrm{bc}$ & $73.6 \pm 4.4 \mathrm{a}$ \\
\hline & & $(10,15)$ & $17.0 \pm 0.4 \mathrm{bcd}$ & $133.5 \pm 10.1 \mathrm{bc}$ & $73.2 \pm 6.3 \mathrm{ab}$ & $26.5 \pm 1.0 \mathrm{ab}$ & $57.6 \pm 7.2 \mathrm{bc}$ \\
\hline & $6: 4$ & $(8,13)$ & $18.2 \pm 0.5 \mathrm{abc}$ & $163.8 \pm 12.0 \mathrm{ab}$ & $61.9 \pm 4.3 \mathrm{~b}$ & $25.0 \pm 0.5 \mathrm{bc}$ & $60.8 \pm 4.0 \mathrm{~b}$ \\
\hline & & $(8,15)$ & $17.0 \pm 0.1 \mathrm{bcd}$ & $154.7 \pm 14.2 \mathrm{abc}$ & $69.1 \pm 4.3 \mathrm{ab}$ & $25.6 \pm 0.9 \mathrm{bc}$ & $53.6 \pm 4.4 \mathrm{c}$ \\
\hline & & $(9,13)$ & $19.8 \pm 0.4 \mathrm{ab}$ & $156.3 \pm 9.2 \mathrm{ab}$ & $67.2 \pm 3.1 \mathrm{ab}$ & $24.0 \pm 1.4 \mathrm{bc}$ & $50.4 \pm 2.6 \mathrm{~cd}$ \\
\hline & & $(9,15)$ & $16.6 \pm 0.1 \mathrm{bcd}$ & $150.8 \pm 12.2 \mathrm{abc}$ & $66.5 \pm 4.5 \mathrm{ab}$ & $26.0 \pm 1.0 \mathrm{ab}$ & $43.2 \pm 3.2 \mathrm{e}$ \\
\hline & & $(10,13)$ & $22.4 \pm 0.8 \mathrm{a}$ & $164.3 \pm 10.6 \mathrm{ab}$ & $68.6 \pm 1.7 \mathrm{ab}$ & $24.4 \pm 0.9 \mathrm{bc}$ & $75.2 \pm 4.1 \mathrm{a}$ \\
\hline & & $(10,15)$ & $17.6 \pm 0.8 \mathrm{bcd}$ & $152.7 \pm 14.6 \mathrm{abc}$ & $62.9 \pm 4.9 \mathrm{~b}$ & $25.5 \pm 1.4 \mathrm{bc}$ & $55.2 \pm 3.6 \mathrm{bc}$ \\
\hline & No & $(0,0)$ & $9.0 \pm 0.5 \mathrm{e}$ & $118.8 \pm 13.4 \mathrm{c}$ & $70.3 \pm 8.8 \mathrm{ab}$ & $27.2 \pm 1.4 \mathrm{a}$ & $23.2 \pm 1.8 \mathrm{f}$ \\
\hline
\end{tabular}

苩肥于 $6 、 7 、 8 、 9$ 叶分别施入, 穗肥于 $11 、 12 、 13 、 14 、 15$ 叶分别施入。 ${ }^{\#}(6,11)$ 组合表示于第 6 叶完全抽出时施入葲肥, 于第 11 叶完全抽出时施入穗肥; 其他依此类推。 $\mathrm{B}: \mathrm{P}$ 为基蒠肥 : 穗肥。相同品种, 同一列中不同小写字母表示在 0.05 水平上差异显著。

Tillering fertilizer was applied when the 6, 7, 8, 9 leaves extracted, panicle fertilizer was applied when the 11,12,13,14,15 leaves extracted. \# $(6,11)$ means that the tillering fertilizer was applied when the sixth leaf was fully extracted, and the panicle fertilizer was applied when the eleventh leaf was completely extracted; the rest may be deduced by analogy. B:P means basal tillering fertilizer: panicle fertilizer. Values within a column followed by different letters are significantly different at $P<0.05$ in the same cultivar.

\section{5 氮素积累量与氮肥利用率}

由表 6 和表 7 可知, 不同氮肥运筹下, 相同(叶 龄模式)施肥处理的氮肥积累量及各氮肥利用率表 现为 $6: 4$ 高于 $8: 2$; 同一氮肥运筹下, 处理吉粳 88-6：4 (8，11)、沈农 265-6：4 (9, 12)、沈农 1401-6：
$4(10,13)$ 的氮素积累总量以及各氮素利用率均显著 高于同品种其他处理, 2 年趋势基本一致。在相同葟 肥施用叶龄下, 3 个水稻品种分别在倒 4 叶穗肥处理 时, 植株的氮素积累总量、吸收利用率、农学利用 率和偏生产力均明显高于倒 2 叶穗肥处理。

表 62016 年不同氮肥运筹下各处理氮素利用率

Table 6 Effects of different nitrogen fertilizers on nitrogen use efficiency in 2016

\begin{tabular}{ccccccc}
\hline $\begin{array}{c}\text { 品种 } \\
\text { Cultivar }\end{array}$ & $\begin{array}{c}\text { 基藮肥:穗肥 } \\
\text { B:P }\end{array}$ & $\begin{array}{c}\text { 处理时期 } \\
\text { Treatment }\end{array}$ & $\begin{array}{c}\text { 每筒氮积累总量 } \\
\text { total uptake } \\
\left(\mathrm{g} \mathrm{pot}^{-1}\right)\end{array}$ & $\begin{array}{c}\text { 吸收利用率 } \\
\text { Recovery } \\
\text { efficiency }(\%)\end{array}$ & $\begin{array}{c}\text { 农学利用率 } \\
\text { Agronomic efficiency } \\
\left(\mathrm{kg} \mathrm{kg} \mathrm{k}^{-1} \mathrm{~N}\right)\end{array}$ & $\begin{array}{c}\text { 偏生产力 } \\
\text { Partial factor } \\
\text { productivity } \\
\left(\mathrm{kg} \mathrm{kg}^{-1} \mathrm{~N}\right)\end{array}$ \\
\hline $\begin{array}{c}\text { 吉粳 } 88 \\
\text { Jigeng } 88\end{array}$ & $8: 2$ & $(6,11)^{\#}$ & $1.22 \pm 0.05 \mathrm{cde}$ & $32.8 \pm 2.3 \mathrm{cde}$ & $20.1 \pm 0.5 \mathrm{~b}$ & $31.5 \pm 0.8 \mathrm{bcd}$ \\
& & $(6,13)$ & $1.15 \pm 0.12 \mathrm{de}$ & $28.8 \pm 7.1 \mathrm{de}$ & $17.6 \pm 0.3 \mathrm{~b}$ & $29.4 \pm 0.6 \mathrm{bcd}$ \\
& $(7,11)$ & $1.28 \pm 0.11 \mathrm{bcd}$ & $36.6 \pm 7.6 \mathrm{bcd}$ & $22.1 \pm 1.8 \mathrm{~b}$ & $33.4 \pm 3.3 \mathrm{abcd}$ \\
& $(7,13)$ & $1.21 \pm 0.02 \mathrm{cde}$ & $32.4 \pm 1.3 \mathrm{cde}$ & $17.6 \pm 2.1 \mathrm{~b}$ & $26.9 \pm 1.9 \mathrm{~cd}$ \\
\hline
\end{tabular}


(续表 6)

\begin{tabular}{|c|c|c|c|c|c|c|}
\hline $\begin{array}{c}\text { 品种 } \\
\text { Cultivar }\end{array}$ & $\begin{array}{c}\text { 基藍肥:穗肥 } \\
\text { B:P }\end{array}$ & $\begin{array}{l}\text { 处理时期 } \\
\text { Treatment }\end{array}$ & $\begin{array}{c}\text { 每筒氮积累总量 } \\
\mathrm{N} \text { total uptake } \\
\left(\mathrm{g} \mathrm{pot}^{-1}\right)\end{array}$ & $\begin{array}{c}\text { 吸收利用率 } \\
\text { Recovery } \\
\text { efficiency (\%) }\end{array}$ & $\begin{array}{c}\text { 农学利用率 } \\
\text { Agronomic efficiency } \\
\left(\mathrm{kg} \mathrm{kg}^{-1} \mathrm{~N}\right)\end{array}$ & $\begin{array}{c}\text { 偏生产力 } \\
\text { Partial factor } \\
\text { productivity } \\
\left(\mathrm{kg} \mathrm{kg}^{-1} \mathrm{~N}\right)\end{array}$ \\
\hline \multirow{9}{*}{$\begin{array}{c}\text { 吉粳 } 88 \\
\text { Jigeng } 88\end{array}$} & & $(8,11)$ & $1.37 \pm 0.10 \mathrm{ab}$ & $42.6 \pm 7.3 \mathrm{ab}$ & $26.7 \pm 3.4 \mathrm{ab}$ & $37.2 \pm 11.9 \mathrm{ab}$ \\
\hline & & $(8,13)$ & $12.30 \pm 0.01 \mathrm{~cd}$ & $33.7 \pm 0.8 \mathrm{cde}$ & $22.6 \pm 2.5 \mathrm{~b}$ & $34.0 \pm 4.6 \mathrm{abc}$ \\
\hline & $6: 4$ & $(6,11)$ & $1.27 \pm 0.04 \mathrm{bcd}$ & $36.2 \pm 3.8 \mathrm{bcd}$ & $23.6 \pm 0.3 \mathrm{ab}$ & $34.8 \pm 0.3 \mathrm{abc}$ \\
\hline & & $(6,13)$ & $1.10 \pm 0.01 \mathrm{e}$ & $25.5 \pm 1.1 \mathrm{e}$ & $18.6 \pm 0.3 \mathrm{~b}$ & $30.1 \pm 0.4 \mathrm{bcd}$ \\
\hline & & $(7,11)$ & $1.42 \pm 0.14 \mathrm{ab}$ & $45.5 \pm 9.9 \mathrm{ab}$ & $25.2 \pm 2.5 \mathrm{~b}$ & $35.8 \pm 4.3 \mathrm{abc}$ \\
\hline & & $(7,13)$ & $1.31 \pm 0.08 \mathrm{abc}$ & $38.7 \pm 5.6 \mathrm{abc}$ & $17.6 \pm 1.8 \mathrm{~b}$ & $28.8 \pm 2.8 \mathrm{bcd}$ \\
\hline & & $(8,11)$ & $1.43 \pm 0.02 \mathrm{a}$ & $45.8 \pm 1.5 \mathrm{a}$ & $32.2 \pm 1.0 \mathrm{a}$ & $42.2 \pm 1.9 \mathrm{a}$ \\
\hline & & $(8,13)$ & $1.16 \pm 0.06 \mathrm{de}$ & $29.2 \pm 4.6 \mathrm{de}$ & $24.1 \pm 0.8 \mathrm{ab}$ & $35.1 \pm 1.3 \mathrm{abc}$ \\
\hline & No & $(0,0)$ & $0.70 \pm 0.02 \mathrm{f}$ & - & - & - \\
\hline \multirow{13}{*}{$\begin{array}{c}\text { 沈农 } 265 \\
\text { Shennong } 265\end{array}$} & $8: 2$ & $(7,12)$ & $1.15 \pm 0.00 \mathrm{~d}$ & $28.1 \pm 0.9 \mathrm{~d}$ & $19.1 \pm 0.8 \mathrm{ef}$ & $36.6 \pm 2.8 \mathrm{~d}$ \\
\hline & & $(7,14)$ & $1.07 \pm 0.01 \mathrm{e}$ & $23.2 \pm 1.6 \mathrm{e}$ & $17.6 \pm 0.3 \mathrm{f}$ & $34.8 \pm 1.9 \mathrm{c}$ \\
\hline & & $(8,12)$ & $1.16 \pm 0.01 \mathrm{~d}$ & $28.9 \pm 2.3 \mathrm{~d}$ & $21.1 \pm 1.0 \mathrm{def}$ & $39.4 \pm 5.9 \mathrm{c}$ \\
\hline & & $(8,14)$ & $1.13 \pm 0.03 \mathrm{~d}$ & $27.3 \pm 0.5 \mathrm{~d}$ & $20.1 \pm 1.5 \mathrm{ef}$ & $38.3 \pm 3.7 \mathrm{c}$ \\
\hline & & $(9,12)$ & $1.36 \pm 0.01 \mathrm{~b}$ & $41.4 \pm 1.3 \mathrm{ab}$ & $27.7 \pm 0.8 \mathrm{~b}$ & $43.5 \pm 3.1 \mathrm{bc}$ \\
\hline & & $(9,14)$ & $1.05 \pm 0.02 \mathrm{e}$ & $21.9 \pm 1.4 \mathrm{e}$ & $21.6 \pm 0.8 \mathrm{def}$ & $39.2 \pm 5.2 \mathrm{bc}$ \\
\hline & $6: 4$ & $(7,12)$ & $1.22 \pm 0.02 \mathrm{c}$ & $32.9 \pm 2.1 \mathrm{c}$ & $21.6 \pm 2.0 \mathrm{de}$ & $39.8 \pm 6.7 \mathrm{bc}$ \\
\hline & & $(7,14)$ & $1.06 \pm 0.02 \mathrm{e}$ & $22.7 \pm 0.5 \mathrm{e}$ & $20.1 \pm 1.0 \mathrm{ef}$ & $35.1 \pm 1.5 \mathrm{bc}$ \\
\hline & & $(8,12)$ & $1.35 \pm 0.02 \mathrm{~b}$ & $40.7 \pm 3.9 \mathrm{~b}$ & $25.7 \pm 1.5 \mathrm{bc}$ & $40.8 \pm 1.9 \mathrm{bc}$ \\
\hline & & $(8,14)$ & $1.16 \pm 0.01 \mathrm{~d}$ & $28.9 \pm 1.6 \mathrm{~d}$ & $23.1 \pm 1.5 \mathrm{cde}$ & $39.0 \pm 2.8 \mathrm{bc}$ \\
\hline & & $(9,12)$ & $1.42 \pm 0.03 \mathrm{a}$ & $45.3 \pm 3.7 \mathrm{a}$ & $31.2 \pm 0.8 \mathrm{a}$ & $46.5 \pm 4.2 \mathrm{abc}$ \\
\hline & & $(9,14)$ & $1.17 \pm 0.06 \mathrm{~d}$ & $29.5 \pm 2.8 \mathrm{~d}$ & $24.6 \pm 1.5 \mathrm{bcd}$ & $38.9 \pm 4.8 \mathrm{ab}$ \\
\hline & No & $(0,0)$ & $0.70 \pm 0.02 \mathrm{f}$ & - & - & - \\
\hline \multirow{13}{*}{$\begin{array}{c}\text { 沈农 } 1401 \\
\text { Shennong } \\
1401\end{array}$} & $8: 2$ & $(8,13)$ & $1.31 \pm 0.09$ bcde & $36.4 \pm 6.2 \mathrm{bcde}$ & $28.7 \pm 2.8 \mathrm{bcd}$ & $36.8 \pm 4.9 \mathrm{bcd}$ \\
\hline & & $(8,15)$ & $1.17 \pm 0.04$ ef & $27.5 \pm 3.3 \mathrm{e}$ & $24.1 \pm 2.3 \mathrm{de}$ & $33.0 \pm 4.1 \mathrm{de}$ \\
\hline & & $(9,13)$ & $1.43 \pm 0.07 \mathrm{ab}$ & $43.9 \pm 5.1 \mathrm{~b}$ & $24.1 \pm 1.8 \mathrm{de}$ & $32.7 \pm 3.1 \mathrm{de}$ \\
\hline & & $(9,15)$ & $1.21 \pm 0.06 \mathrm{ef}$ & $29.7 \pm 4.2 \mathrm{de}$ & $21.6 \pm 1.5 \mathrm{e}$ & $30.5 \pm 2.6 \mathrm{e}$ \\
\hline & & $(10,13)$ & $1.42 \pm 0.07 \mathrm{ab}$ & $43.2 \pm 4.9 \mathrm{~b}$ & $30.2 \pm 2.3 \mathrm{abc}$ & $38.4 \pm 4.1 \mathrm{abc}$ \\
\hline & & $(10,15)$ & $1.24 \pm 0.07 \mathrm{cdef}$ & $31.9 \pm 5.2 \mathrm{cde}$ & $23.1 \pm 2.0 \mathrm{de}$ & $32.2 \pm 3.4 \mathrm{de}$ \\
\hline & $6: 4$ & $(8,13)$ & $1.39 \pm 0.16 \mathrm{abc}$ & $41.2 \pm 10.6 \mathrm{bc}$ & $32.2 \pm 1.5 \mathrm{ab}$ & $41.2 \pm 1.0 \mathrm{ab}$ \\
\hline & & $(8,15)$ & $1.23 \pm 0.02 \mathrm{def}$ & $31.1 \pm 2.3 \mathrm{cde}$ & $26.2 \pm 0.5 \mathrm{cde}$ & $34.5 \pm 1.1 \mathrm{cde}$ \\
\hline & & $(9,13)$ & $1.44 \pm 0.12 \mathrm{ab}$ & $44.3 \pm 8.4 \mathrm{ab}$ & $27.2 \pm 0.8 \mathrm{bcd}$ & $35.2 \pm 1.2 \mathrm{cde}$ \\
\hline & & $(9,15)$ & $1.16 \pm 0.05 \mathrm{f}$ & $26.6 \pm 3.6 \mathrm{e}$ & $24.6 \pm 1.3 \mathrm{de}$ & $33.3 \pm 2.2 \mathrm{de}$ \\
\hline & & $(10,13)$ & $1.47 \pm 0.11 \mathrm{a}$ & $46.2 \pm 7.2 \mathrm{a}$ & $34.7 \pm 1.0 \mathrm{a}$ & $43.2 \pm 1.4 \mathrm{a}$ \\
\hline & & $(10,15)$ & $1.36 \pm 0.06 \mathrm{abcd}$ & $39.4 \pm 4.6 \mathrm{bcd}$ & $24.1 \pm 1.0 \mathrm{de}$ & $32.9 \pm 1.8 \mathrm{de}$ \\
\hline & No & $(0,0)$ & $0.73 \pm 0.04 \mathrm{~g}$ & - & - & - \\
\hline
\end{tabular}

菜肥于 $6 、 7 、 8 、 9$ 叶分别施入, 穗肥于 $11 、 12 、 13 、 14 、 15$ 叶分别施入。 ${ }^{\#}(6,11)$ 组合表示于第 6 叶完全抽出时施入毷肥, 于第 11 叶完全抽出时施入穗肥; 其他依此类推。 $\mathrm{B}: \mathrm{P}$ 为基菜肥 : 穗肥。相同品种, 同一列中不同小写字母表示在 0.05 水平上差异显著。

Tillering fertilizer was applied when the 6, 7, 8, 9 leaves extracted, panicle fertilizer was applied when the 11, 12, 13, 14, 15 leaves extracted. \# $(6,11)$ means that the tillering fertilizer was applied when the sixth leaf was fully extracted, and the panicle fertilizer was applied when the eleventh leaf was completely extracted; the rest may be deduced by analogy. B:P means basal tillering fertilizer: panicle fertilizer. Values within a column followed by different letters are significantly different at $P<0.05$ in the same cultivar. 
表 72017 年不同氮肥运筹下各处理氮素利用率

Table 7 Effects of different nitrogen fertilizers on nitrogen use efficiency in 2017

\begin{tabular}{|c|c|c|c|c|c|c|}
\hline $\begin{array}{c}\text { 品种 } \\
\text { Cultivar }\end{array}$ & $\begin{array}{c}\text { 基藍肥:穗肥 } \\
\text { B:P }\end{array}$ & $\begin{array}{l}\text { 处理时期 } \\
\text { Treatment }\end{array}$ & $\begin{array}{c}\text { 每筒氮积累总量 } \\
\mathrm{N} \text { total uptake } \\
\left(\mathrm{g} \mathrm{pot}^{-1}\right)\end{array}$ & $\begin{array}{c}\text { 吸收利用率 } \\
\text { Recovery efficiency } \\
(\%)\end{array}$ & $\begin{array}{c}\text { 农学利用率 } \\
\text { Agronomic efficiency } \\
\left(\mathrm{kg} \mathrm{kg}^{-1} \mathrm{~N}\right)\end{array}$ & $\begin{array}{c}\text { 偏生产力 } \\
\text { Partial factor } \\
\text { productivity } \\
\left(\mathrm{kg} \mathrm{kg}^{-1} \mathrm{~N}\right)\end{array}$ \\
\hline \multirow{13}{*}{$\begin{array}{c}\text { 吉粳 } 88 \\
\text { Jigeng } 88\end{array}$} & \multirow[t]{6}{*}{$8: 2$} & $(6,11)^{\#}$ & $1.10 \pm 0.07 \mathrm{de}$ & $21.8 \pm 0.7 \mathrm{bcd}$ & $25.2 \pm 3.3 \mathrm{abc}$ & $36.2 \pm 2.1 \mathrm{~b}$ \\
\hline & & $(6,13)$ & $1.02 \pm 0.06 \mathrm{e}$ & $16.8 \pm 0.8 \mathrm{~d}$ & $20.1 \pm 1.5 \mathrm{bc}$ & $36.0 \pm 2.4 \mathrm{~b}$ \\
\hline & & $(7,11)$ & $1.28 \pm 0.03 \mathrm{bc}$ & $30.4 \pm 6.2 \mathrm{abc}$ & $29.2 \pm 2.0 \mathrm{abc}$ & $37.3 \pm 2.6 \mathrm{~b}$ \\
\hline & & $(7,13)$ & $1.10 \pm 0.06 \mathrm{de}$ & $19.0 \pm 13.7 \mathrm{~cd}$ & $21.6 \pm 2.0 \mathrm{abc}$ & $36.2 \pm 1.2 \mathrm{~b}$ \\
\hline & & $(8,11)$ & $1.29 \pm 0.04 \mathrm{bc}$ & $30.7 \pm 6.6 \mathrm{abc}$ & $36.2 \pm 3.5 \mathrm{a}$ & $41.4 \pm 2.3 \mathrm{ab}$ \\
\hline & & $(8,13)$ & $1.26 \pm 0.05 \mathrm{c}$ & $28.9 \pm 7.4 \mathrm{abcd}$ & $30.2 \pm 2.4 \mathrm{abc}$ & $36.3 \pm 1.7 \mathrm{~b}$ \\
\hline & \multirow[t]{6}{*}{$6: 4$} & $(6,11)$ & $1.32 \pm 0.05 \mathrm{bc}$ & $35.3 \pm 0.8 \mathrm{a}$ & $25.7 \pm 2.3 \mathrm{abc}$ & $36.4 \pm 2.9 \mathrm{~b}$ \\
\hline & & $(6,13)$ & $1.03 \pm 0.04 \mathrm{e}$ & $17.0 \pm 1.5 \mathrm{~d}$ & $20.6 \pm 0.8 \mathrm{abc}$ & $37.4 \pm 2.3 \mathrm{~b}$ \\
\hline & & $(7,11)$ & $1.33 \pm 0.03 \mathrm{abc}$ & $33.3 \pm 5.5 \mathrm{ab}$ & $25.2 \pm 3.3 \mathrm{abc}$ & $43.5 \pm 2.2 \mathrm{ab}$ \\
\hline & & $(7,13)$ & $1.15 \pm 0.07 \mathrm{~d}$ & $22.1 \pm 13.5 \mathrm{bcd}$ & $27.7 \pm 3.0 \mathrm{abc}$ & $40.5 \pm 3.7 \mathrm{ab}$ \\
\hline & & $(8,11)$ & $1.42 \pm 0.03 \mathrm{a}$ & $38.7 \pm 1.8 \mathrm{a}$ & $36.7 \pm 4.0 \mathrm{a}$ & $47.2 \pm 1.4 \mathrm{a}$ \\
\hline & & $(8,13)$ & $1.37 \pm 0.05 \mathrm{ab}$ & $35.5 \pm 4.9 \mathrm{a}$ & $29.2 \pm 0.8 \mathrm{abc}$ & $42.3 \pm 2.6 \mathrm{ab}$ \\
\hline & N0 & $(0,0)$ & $0.76 \pm 0.06 \mathrm{f}$ & - & - & - \\
\hline \multirow{13}{*}{$\begin{array}{c}\text { 沈农 } 265 \\
\text { Shennong } 265\end{array}$} & \multirow[t]{6}{*}{$8: 2$} & $(7,12)$ & $1.21 \pm 0.05 \mathrm{~d}$ & $28.3 \pm 2.7 \mathrm{~d}$ & $24.1 \pm 5.0 \mathrm{bc}$ & $39.5 \pm 15.2 \mathrm{de}$ \\
\hline & & $(7,14)$ & $1.18 \pm 0.05 \mathrm{~d}$ & $26.9 \pm 1.3 \mathrm{~d}$ & $16.6 \pm 2.3 \mathrm{~d}$ & $33.1 \pm 6.5 \mathrm{e}$ \\
\hline & & $(8,12)$ & $1.46 \pm 0.04 \mathrm{ab}$ & $44.1 \pm 2.3 \mathrm{ab}$ & $25.2 \pm 3.5 \mathrm{bc}$ & $41.4 \pm 4.3 \mathrm{cde}$ \\
\hline & & $(8,14)$ & $1.30 \pm 0.04 \mathrm{c}$ & $34.4 \pm 2.7 \mathrm{c}$ & $17.1 \pm 2.1 \mathrm{~d}$ & $34.9 \pm 6.7 \mathrm{de}$ \\
\hline & & $(9,12)$ & $1.47 \pm 0.01 \mathrm{ab}$ & $45.0 \pm 1.6 \mathrm{ab}$ & $28.2 \pm 2.9 \mathrm{ab}$ & $47.8 \pm 5.5 \mathrm{ab}$ \\
\hline & & $(9,14)$ & $1.42 \pm 0.04 \mathrm{~b}$ & $41.5 \pm 1.2 \mathrm{~b}$ & $21.1 \pm 2.1 \mathrm{~cd}$ & $38.4 \pm 7.3 \mathrm{de}$ \\
\hline & \multirow[t]{6}{*}{$6: 4$} & $(7,12)$ & $1.43 \pm 0.03 \mathrm{ab}$ & $42.3 \pm 3.5 \mathrm{ab}$ & $26.7 \pm 2.6 \mathrm{~b}$ & $42.5 \pm 5.2 \mathrm{bcd}$ \\
\hline & & $(7,14)$ & $1.22 \pm 0.01 \mathrm{~d}$ & $29.0 \pm 1.6 \mathrm{~d}$ & $23.1 \pm 2.6 \mathrm{~cd}$ & $38.4 \pm 7.5 \mathrm{de}$ \\
\hline & & $(8,12)$ & $1.47 \pm 0.05 \mathrm{ab}$ & $44.7 \pm 4.1 \mathrm{ab}$ & $26.7 \pm 2.3 \mathrm{~b}$ & $33.3 \pm 19.0 \mathrm{de}$ \\
\hline & & $(8,14)$ & $1.41 \pm 0.04 \mathrm{~b}$ & $41.0 \pm 3.0 \mathrm{~b}$ & $23.1 \pm 2.5 \mathrm{~cd}$ & $39.0 \pm 5.4 \mathrm{de}$ \\
\hline & & $(9,12)$ & $1.49 \pm 0.04 \mathrm{a}$ & $46.3 \pm 2.9 \mathrm{a}$ & $29.7 \pm 3.6 \mathrm{a}$ & $43.8 \pm 11.5 \mathrm{bc}$ \\
\hline & & $(9,14)$ & $1.48 \pm 0.00 \mathrm{ab}$ & $45.3 \pm 2.2 \mathrm{ab}$ & $21.6 \pm 3.4 \mathrm{~cd}$ & $38.2 \pm 9.9 \mathrm{de}$ \\
\hline & N0 & $(0,0)$ & $0.76 \pm 0.04 \mathrm{e}$ & - & - & - \\
\hline \multirow{10}{*}{$\begin{array}{c}\text { 沈农 } 1401 \\
\text { Shennong } \\
1401\end{array}$} & \multirow[t]{6}{*}{$8: 2$} & $(8,13)$ & $1.15 \pm 0.14 \mathrm{efg}$ & $25.2 \pm 8.9$ efg & $20.6 \pm 1.5 \mathrm{bc}$ & $27.9 \pm 5.8 \mathrm{abcd}$ \\
\hline & & $(8,15)$ & $1.00 \pm 0.08 \mathrm{~g}$ & $15.6 \pm 5.0 \mathrm{~h}$ & $15.6 \pm 2.5 \mathrm{de}$ & $25.8 \pm 2.0 \mathrm{~cd}$ \\
\hline & & $(9,13)$ & $1.16 \pm 0.09 \mathrm{ef}$ & $25.6 \pm 5.5 \mathrm{ef}$ & $24.1 \pm 2.6 \mathrm{~b}$ & $41.7 \pm 18.1 \mathrm{ab}$ \\
\hline & & $(9,15)$ & $1.02 \pm 0.10 \mathrm{fg}$ & $16.5 \pm 6.4 \mathrm{~g}$ & $11.6 \pm 2.3 \mathrm{e}$ & $30.8 \pm 3.9 \mathrm{abcd}$ \\
\hline & & $(10,13)$ & $1.49 \pm 0.04 \mathrm{ab}$ & $46.3 \pm 2.4 \mathrm{ab}$ & $31.7 \pm 2.8 \mathrm{a}$ & $40.9 \pm 5.8 \mathrm{ab}$ \\
\hline & & $(10,15)$ & $1.20 \pm 0.09 \mathrm{de}$ & $28.3 \pm 5.8 \mathrm{de}$ & $21.6 \pm 4.5 \mathrm{bc}$ & $37.5 \pm 11.2 \mathrm{abc}$ \\
\hline & \multirow[t]{4}{*}{$6: 4$} & $(8,13)$ & $1.39 \pm 0.08 \mathrm{abc}$ & $40.3 \pm 4.9 \mathrm{abc}$ & $23.6 \pm 2.5 \mathrm{~b}$ & $34.4 \pm 13.0 \mathrm{abc}$ \\
\hline & & $(8,15)$ & $1.30 \pm 0.13 \mathrm{cde}$ & $34.4 \pm 8.2$ cde & $19.1 \pm 2.8 \mathrm{c}$ & $27.5 \pm 0.6 \mathrm{bcd}$ \\
\hline & & $(9,13)$ & $1.51 \pm 0.06 \mathrm{a}$ & $47.6 \pm 3.6 \mathrm{a}$ & $17.1 \pm 1.6 \mathrm{~cd}$ & $41.0 \pm 9.8 \mathrm{a}$ \\
\hline & & $(9,15)$ & $1.28 \pm 0.06 \mathrm{cde}$ & $33.1 \pm 3.8 \mathrm{cde}$ & $12.6 \pm 2.0 \mathrm{e}$ & $32.0 \pm 8.1 \mathrm{abcd}$ \\
\hline
\end{tabular}




\begin{tabular}{|c|c|c|c|c|c|c|}
\hline $\begin{array}{c}\text { 品种 } \\
\text { Cultivar }\end{array}$ & $\begin{array}{c}\text { 基蒒肥:穗肥 } \\
\text { B:P }\end{array}$ & $\begin{array}{l}\text { 处理时期 } \\
\text { Treatment }\end{array}$ & $\begin{array}{l}\text { 每筒氮积累总量 } \\
\mathrm{N} \text { total uptake } \\
\left(\mathrm{g} \mathrm{pot}^{-1}\right)\end{array}$ & $\begin{array}{c}\text { 吸收利用率 } \\
\text { Recovery efficiency } \\
(\%)\end{array}$ & $\begin{array}{c}\text { 农学利用率 } \\
\text { Agronomic efficiency } \\
\left(\mathrm{kg} \mathrm{kg}^{-1} \mathrm{~N}\right)\end{array}$ & $\begin{array}{c}\text { 偏生产力 } \\
\text { Partial factor } \\
\text { productivity } \\
\left(\mathrm{kg} \mathrm{kg}^{-1} \mathrm{~N}\right)\end{array}$ \\
\hline 沈农 1401 & & $(10,13)$ & $1.53 \pm 0.03 \mathrm{a}$ & $48.8 \pm 1.6 \mathrm{a}$ & $32.7 \pm 2.6 \mathrm{a}$ & $41.9 \pm 8.4 \mathrm{a}$ \\
\hline Shennong & & $(10,15)$ & $1.35 \pm 0.12 \mathrm{bcd}$ & $37.5 \pm 7.3 \mathrm{bcd}$ & $20.1 \pm 2.3 \mathrm{bc}$ & $28.2 \pm 4.6 \mathrm{abcd}$ \\
\hline 1401 & No & $(0,0)$ & $0.77 \pm 0.02 \mathrm{~h}$ & - & - & - \\
\hline
\end{tabular}

藍肥于 $6 、 7 、 8 、 9$ 叶分别施入, 穗肥于 $11 、 12 、 13 、 14 、 15$ 叶分别施入。 ${ }^{*}(6,11)$ 组合表示于第 6 叶完全抽出时施入菜肥, 于第 11 叶完全抽出时施入穗肥; 其他依此类推。 $\mathrm{B}: \mathrm{P}$ 为基蔡肥：穗肥。相同品种, 同一列中不同小写字母表示在 0.05 水平上差异显著。

Tillering fertilizer was applied when the 6,7,8,9 leaves extracted, panicle fertilizer was applied when the 11,12,13,14, 15 leaves extracted. \# $(6,11)$ means that the tillering fertilizer was applied when the sixth leaf was fully extracted, and the panicle fertilizer was applied when the eleventh leaf was completely extracted; the rest may be deduced by analogy. B:P means basal tillering fertilizer: panicle fertilizer. Values within a column followed by different letters are significantly different at $P<0.05$ in the same cultivar.

\section{3 讨论}

3.1 不同叶龄藩、穗氮肥组合对粳稻穗数、颖花 数和产量的影响

单位面积颖花量是穗数和每穗粒数的乘积, 单 位面积颖花数越多越有利于产量的提高 ${ }^{[18]}$, 而每穗 颖花数的多少决定于分化颖花数和退化颖花数 ${ }^{[19]}$ 。 杨弘远等 ${ }^{[20]}$ 研究表明, 在幼穗发育的前期就应促使 颖花形成, 降低颖花退化, 确保每穗颖花数的增多。 张洪程等 ${ }^{[21]}$ 、胡群等 ${ }^{[22]}$ 、严田蓉等 ${ }^{[23]}$ 研究认为, 高 产水稻在穗期吸氮较多, 应适当降低前期基藍肥用 量, 增加后期穗粒肥用量, 使基菜肥与穗粒肥配比 达 6：4, 以优化整个水稻生育期的群体质量, 同时 能提高每穗粒数、结实率和千粒重。本文研究结果 表明, 不同叶龄藍肥、穗肥组合处理下, 基藍肥与 穗肥比例为 $6: 4$ 时, 各处理分化颖花数和实际颖 花数均显著高于 8：2, 但退化颖花数也相应增加, 这可能与总颖花量大幅提高后源、库协调关系发生 变化、源相对不足有关, 也可能与自身的环境适应 能力有关。

合理氮肥运筹是影响分葟成穗率和产量最重要 的因素, 穗肥能增加每穗颖花数, 提高结实率和千 粒重, 其中穗肥最佳施用叶龄期为倒 4 叶和倒 2 叶 两个时期 ${ }^{[24-25]}$ 。丁艳锋等 ${ }^{[26]}$ 在研究不同穗肥施用时 期时发现, 在较早穗肥施用时, 较大穗型水稻品种 的每穗粒数明显增加, 从而产量也明显增加, 而小 穗型品种表现为结实率明显提高导致产量增加。本 试验结果显示, 3 个大穗型粳稻品种吉粳 88 、沈农 265 和沈农 1401 均在倒 4 叶施入穗肥时明显比倒 2 叶施入穗肥时的分化颖花数和实际颖花数高, 且产 量最高, 这与超高产水稻应是沿着“足额的穗数 $\rightarrow$
较多的总颖花数 $\rightarrow$ 巨大的库容量 $\rightarrow$ 超高产” 这一主 线推进 ${ }^{[27]}$ 相一致。

水稻藍肥是决定足额穗数的前提保证。在美 国加利福尼亚州的灌溉水稻生产中, 菜肥施用时 期通常在最大分竪期 ${ }^{[28]}$ 。在我国南方广东等地区 采用的水稻“三控”施肥技术中，藍肥施用时期在 分葍中期 ${ }^{[29]}$ 。黄大山等 ${ }^{[30]}$ 在研究不同时期施用分薛 肥时, 发现与常规移栽后 5 7 d 施入萆肥处理相比, 4 种早稻或晚稻品种均在移栽后 $14 \mathrm{~d}$ 左右施入菜肥 成穗率显著提高。与传统栽培不同的是, 本试验采 取水稻分竪期不进行控藍处理, 并且在分藍高峰期

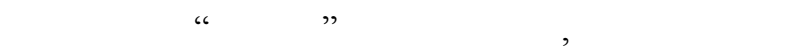
界期前形成的中小蒒萃能保藍成穗，而在其后新增的 小菜能促藍增穗，总体成穗率相应提高。本研究櫱、 穗叶龄氮肥组合结果表明, 在基葍肥与穗肥运筹比 例 6：4 基础上, 菜肥适当后移(保藍肥), 避免了因 韸肥过早施入或施入过量造成前期浪费、后期脱肥, 有效分蒒临界期已有茎䔣营养不足而成穗小，新增 小藍营养不足而难成穗现象的发生; 穗肥适当前移 (促花肥), 避免了因穗肥过晚施入造成颖花分化不 足，库容量降低。蒠、穗叶龄合理组合施氮符合水 稻营养生长和幼穗发育期间对氮素的吸收利用规 律, 能够起到增加有效穗数和提高库容量的作用, 从而更有利于增产, 这与本课题组前期研究结果一 致 ${ }^{[31]}$ 。此外, 氮肥施用量对水稻产量及农艺性状的 影响因品种不同而有所差异，本试验仅在中量供氮 水平下, 研究了 3 个不同叶片数粳稻直立或半直立 穗型品种的叶龄藮、穗氮肥组合对粳稻茎藮动态、 成穗率、颖花分化及产量构成因素的影响, 而关于 不同生育类型粳稻品种在不同供氮水平及田间配置 下茎藍成穗、颖花分化及产量构成的变化规律，是 
否符合本研究结果, 还需进一步验证。

\section{2 不同叶龄蒏、穗氮肥组合对粳稻氮素利用效} 率的影响

我国衡量氮肥利用率的一个指标主要是氮肥吸 收利用率。杨海生等 ${ }^{[32]}$ 认为依叶龄追施氮肥能稳产 高产, 提高氮效率。林忠成等 ${ }^{[33}$ 和许轫等 ${ }^{\left[{ }^{[3]}\right.}$ 在研究 双季粳稻时, 发现双季稻区的基菜肥与穗肥为 $6: 4$ 时水稻氮素利用率显著高于 8：2。Wang 等 ${ }^{[35]}$ 认为 降低基藍肥比例, 适当推迟藍肥的追肥时间, 可提 高氮肥利用率。张洪程等 ${ }^{[12]}$ 认为在水稻倒 4 、倒 3 叶期施用穗肥, 水稻植株氮素积累量和氮素利用率 最高, 利于高产稳产。刘立军等 ${ }^{[36]}$ 按照实地氮肥管 理方式对汕优 63 施肥, 发现在移栽后 15 19 d 施荠 肥, 水稻的氮肥利用率显著提高。本研究也认为适 当推迟菜肥施用时期可提高氮肥利用率, 粳稻进入 最高分櫱期前后, 即吉粳 88 在 8 叶期(叶龄指数 $57.1 \%$ ), 沈农 265 在 9 叶期(叶龄指数 $60.0 \%$ ), 沈农 1401 在 10 叶期(叶龄指数 $62.5 \%$ )时施用藮肥, 其保 薛成穗、促菜增穗效果要优于分蒒快速增长始期和 分萻快速增长期, 氮肥利用效率显著提高。而在相 同基䔉肥比例下，不同品种诸处理穗肥均以穗分化 始期(倒四叶)促花效果最佳, 即吉粳 88 于 11 叶(叶 龄指数 $78.6 \%$ ), 沈农 265 于 12 叶(叶龄指数 $80.0 \%$ ), 沈农 1401 于 13 叶(叶龄指数 $81.3 \%$ )施用穗肥时, 氮 素积累量、农学利用率以及偏生产力等较高。

本研究采取田间筒栽方式, 既有小筒试验的精 确性、可控性, 又有大田试验的开放性、普适性, 各 筒内部上下通透而周边封闭, 筒间集群而形成群体 生长环境, 因而在施肥处理上可以精确控制, 而土 壤和生态环境又可以最大限度地与田间小区接近, 这就在一定程度上大大提升了试验结果的精准性和 可靠性。但尽管如此, 由于试验结果(尤其是产量结 果) 是以单筒或单穴来统计的, 终非小区群体数据, 而且在试验样本数量、代表性、田间生长状态等方 面, 毕竟与大田小区试验不同, 因此, 相关试验结 果、结论等还需在群体条件下进一步印证。此外, 本 试验年际间的气候差异明显, 影响了个别试验结果 的年际间稳定性, 如 2016 年水稻灌浆期温度较高, 影响了水稻籽粒的灌浆结实及氮素利用率, 这也是 需要注意的。

综上所述, 在水稻氮肥施用上, 应确保适宜 的施氮量, 优化氮肥施用比例和施用时期, 协调 营养生长与生殖生长, 促进源、库、流三者高位平
衡, 以获得最佳产量构成因素, 进而实现增产增 效双重目标。

\section{4 结论}

基葟肥与穗肥运筹比例 $6: 4$ 显著优于 $8: 2$, 且 高产最适宜的叶龄组合为吉粳 88-6：4 $(8,11)$ 、沈农 265-6：4 (9，12)、沈农 1401-6：4 (10,13), 即提高穗 肥比例、葟肥施用时期后移(叶龄指数在 $60.0 \%$ 左右), 穗肥施用时期前移(叶龄指数在 $80.0 \%$ 左右), 能显著 促进有效分菜和分化颖花数的提高, 起到保藍促花 效果, 显著提高氮素积累量、氮素吸收利用率、农 学利用率和偏生产力, 最终达到高产高效的目的。

\section{References}

[1] 孙永健, 孙园园, 蒋明金, 李应洪, 严奉君, 徐徽, 王海月, 马 均. 施肥水平对不同氮效率水稻氮素利用特征及产量的影响. 中国农业科学, 2016, 49: 4745-4756.

Sun Y J, Sun Y Y, Jiang M J, Li Y H, Yan F J, Xu H, Wang H Y, Ma J. Effects of fertilizer levels on nitrogen utilization characterristics and yield in rice cultivars with different nitrogen use efficiencies. Sci Agric Sin, 2016, 49: 4745-4756 (in Chinese with English abstract).

[2] Zhong X H, Peng S B, Buresh R J, Huang J L, Yang J C, Zou Y B, Zhang X H, Wang G H, Zhang F S. Strategies for overcoming low agronomic nitrogen use efficiency in irrigated rice systems in China. Field Crops Res, 2006, 96: 37-47.

[3] 范立慧, 徐珊珊, 侯朋福, 薛利红, 李刚华, 丁艳锋, 杨林章. 不同地力下基蓝肥运筹比例对水稻产量及氮肥吸收利用的影 响. 中国农业科学, 2016, 49: 1872-1884.

Fan L H, Xu S S, Hou P F, Xue L H, Li G H, Ding Y F, Yang L Z. Effect of different ratios of basal to tiller nitrogen on rice yield and nitrogen utilization under different soil fertility. Sci Agric Sin, 2016, 49: 1872-1884 (in Chinese with English abstract).

[4] Peng S B, Huang J L, Zhong X H, Yang J C, Wang G H, Zou Y B, Zhang F S, Zhu Q S, Buresh R, Witt C. Challenge and opportunity in improving fertilizer-nitrogen use efficiency of irrigated rice in China. J Integr Agric, 2002, 1: 776-785.

[5] 罗文姬, 江立庚, 覃丽玲, 梁天锋, 罗必敬. 氮肥基追比对机 插稻产量和氮肥利用效率的影响. 作物杂志, 2016, 32(5): 119-123.

Luo W J, Jiang L G, Qin L L, Liang T F, Luo B J. Nitrogen fertilization ratio on grain yield and nitrogen use efficiency of mechanical transplanting rice. Crops, 2006, 32(5): 119-123 (in Chinese with English abstract).

[6] 白志刚, 张均华, 黄洁, 朱练峰, 曹小闯, 朱春权, 钟楚, 金千 瑜. 氮肥运筹对水稻氮代谢及稻田土壤氮素迁移转化的影响. 生态学杂志, 2018, 37: 3440-3448.

Bai Z G, Zhang J H, Huang J, Zhu L F, Cao X C, Zhu C Q, Zhong $\mathrm{C}$, Jin $\mathrm{Q}$ Y. Effects of nitrogen regime on nitrogen metabolism of rice and nitrogen transformation and translocation in paddy soils. Chin J Ecol, 2018, 37: 3440-3448 (in Chinese with English abstract). 
[7] 霍中洋, 魏海燕, 张洪程, 龚振恺, 戴其根, 许轫. 穗肥运筹 对不同秧龄机插超级稻宁粳 1 号产量及群体质量的影响. 作 物学报, 2012, 38: 1460-1470.

Huo Z Y, Wei H Y, Zhang H C, Gong Z K, Dai Q G, Xu K. Effect of panicle nitrogen fertilizer management on yield and population quality in mechanical transplanted super rice Ningjing 1 with different seedling ages. Acta Agron Sin, 2012, 38: 1460-1470 (in Chinese with English abstract).

[8] 丁艳锋, 刘胜环, 王绍华, 王强盛, 黄丕生, 凌启鸿. 氮素基、 藍肥用量对水稻氮素吸收与利用的影响. 作物学报, 2004, 30(8): 739-744.

Ding Y F, Liu S H, Wang S H, Wang Q S, Huang P S, Ling Q H. Effects of amount of basic and tillering nitrogen applied on absorption and utilization of nitrogen in rice. Acta Agron Sin, 2004, 30: 739-744 (in Chinese with English abstract).

[9] 王维金, 徐竹生, 鲍隆清. 重施穗肥对杂交水稻的产量和氮素 营养的影响. 华中农业大学学报, 1993, 12(3): 209-214.

Wang W J, Xu Z S, Bao L Q. Effects of heavy spike fertilizer on yield and nitrogen in middle-season hybrid rice. J Huazhong Agric Univ, 1993, 12(3): 209-214 (in Chinese with English abstract).

[10] 薛亚光, 陈婷婷, 杨成, 王志琴, 刘立军, 杨建昌. 中粳稻不 同栽培模式对产量及其生理特性的影响. 作物学报, 2010, 36: 466-476.

Xue Y G, Chen T T, Cheng Y, Wang Z Q, Liu L J, Yang J C. Effects of different cultivation patterns on the yield and physiological characteristics in mid-season japonica rice. Acta Agron Sin, 2010, 36: 466-476 (in Chinese with English abstract).

[11] 凌启鸿, 张洪程, 戴其根, 丁艳锋, 凌励, 苏祖芳, 徐茂, 网金 华, 王绍华. 水稻精确定量施氮研究. 中国农业科学, 2005, 38: 2457-2467.

Ling Q H, Zhang H C, Dai Q G, Ding Y F, Ling L, Su Z F, Xu M, Que J H, Wang S H. Study on precise and quantitative N application in rice. Sci Agric Sin, 2005, 38: 2457-2467 (in Chinese with English abstract).

[12] 张洪程, 吴桂成, 戴其根, 霍忠洋, 许轫, 高辉, 魏海燕, 吕修 涛, 万鉦军, 黄银忠. 水稻氮肥精确后移及其机制. 作物学报, 2011, 37: 1837-1851.

Zhang H C, Wu G C, Dai Q G, Huo Z Y, Xu K, Gao H, Wei H Y, Lyu X T, Wan L J, Huang Y Z. Precise postponing nitrogen application and its mechanism in rice. Acta Agron Sin, 2011, 37: 1837-1851 (in Chinese with English abstract).

[13] 凌启鸿, 张洪程, 苏祖芳, 凌励. 稻作新理论: 水稻叶龄模式. 北京: 科学出版社, 1994.

Ling Q H, Zhang H C, Su Z F, Ling L. New Theory of Rice Cultivation: the Leaf-age-model of Rice. Beijing: Science Press, 1994 (in Chinese).

[14] 凌启鸿, 张洪程, 丁艳锋, 戴其根, 凌励, 王绍华, 徐茂. 水稻 高产精确定量栽培. 北方水稻, 2007, 37(2): 1-9.

Ling Q H, Zhang H C, Ding Y F, Dai Q G, Ling L, Wang S H, Xu M. Precise and quantitative cultivation for high yield in rice. North Rice, 2007, 37(2): 1-9 (in Chinese with English abstract).

[15] 张洪程, 吴桂成, 吴文革, 戴其根, 霍忠洋, 许轲, 高辉, 魏海 燕, 黄幸福, 龚金龙. 水稻“精苗稳前、控藮优中、大穗强后” 超高产定量化栽培模式. 中国农业科学, 2010, 43: 2645-2660. Zhang H C, Wu G C, Wu W G, Dai Q G, Huo Z Y, Xu K, Gao H,
Wei H Y, Huang X F, Gong J L. The SOI model of quantitative cultivation of super-high yielding rice. Sci Agric Sin, 2010, 43: 2645-2660 (in Chinese with English abstract).

[16] 万生军, 张洪程, 霍中洋, 林忠成, 戴其根, 许轫, 张军. 氮肥 运筹对超级杂交粳稻产量、品质及氮素利用率的影响. 作物学 报, 2007, 33: 175-182.

Wang L J, Zhang H C, Huo Z Y, Lin Z C, Dai Q G, Xu K, Zhang J. Effects of nitrogen application regimes on yield, quality, and nitrogen use efficiency of super japonica hybrid rice. Acta Agron Sin, 2007, 33: 175-182 (in Chinese with English abstract).

[17] 柳新伟, 孟亚利, 周治国, 曹卫星. 水稻颖花分化与退化的动 态特征. 作物学报, 2005, 31: 451-455.

Liu X W, Meng Y L, Zhou Z G, Cao W X. Dynamic characteristics of floret differentiation and degeneration in rice. Acta Agron Sin, 2005, 31: 451-455 (in Chinese with English abstract).

[18] Kim H Y, Lieffering M, Miura S, Kobayashi K, Okada M. Growth and nitrogen uptake of $\mathrm{CO}_{2}$-enriched rice under field conditions. New Phytol, 2001, 150: 223-229.

[19] 杨洪建, 杨连新, 刘红江, 董桂春, 颜士敏, 朱建国, 王余龙. FACE 对武香粳 14 根系活性影响的研究. 作物学报, 2006, 32(1): 118-124.

Yang H J, Yang L X, Huang J Y, Liu H J, Dong G C, Yan S M, Zhu J G, Wang Y L. Effect of free-air $\mathrm{CO}_{2}$ enrichment on spikelet differentiation and degeneration of japonica rice (Oryza sativa L.) cultivar Wuxiangjing 14. Acta Agron Sin, 2006, 32: 118-124 (in Chinese with English abstract).

[20] 杨弘远. 水稻生殖生物学. 杭州: 浙江大学出版社, 2005. Yang H Y. Rice Reproductive Biological. Hangzhou: Zhejiang University Press, 2005 (in Chinese).

[21] 张洪程, 戴其根, 霍中洋, 许轫, 高辉, 魏海燕, 桂玉清, 吴文 革, 吴桂成, 端木银熙, 孙菊英, 赵品恒, 徐军, 李杰, 王艳, 龚金龙, 姚义, 沙安勤, 周有炎, 李德剑, 肖跃成, 王宝金, 吴 爱国, 钱宗华, 淤永杰, 李华. 水稻超高产栽培研究与探讨. 中国稻米, 2012, 18(1): 1-14.

Zhang H C, Dai Q G, Huo Z Y, Xu K, Gao H, Wei H Y, Gui Y Q, Wu W G, Wu G C, Duan-Mu Y X, Sun J Y, Zhao P H, Xu J, Li J, Wang Y, Gong J L, Yao Y, Sha A Q, Zhou Y Y, Li D J, Xiao Y C, Wang B J, Wu A G, Qian Z H, Yu Y J. Li H. Research and discussion on super high yielding cultivation of rice. Chin Rice, 2012, 18(1): 1-14 (in Chinese with English abstract).

[22] 胡群, 夏敏, 张洪程, 曹利强, 郭保卫, 魏海燕, 陈厚存, 戴其 根, 霍中洋, 许轫, 林昌明, 韩宝富. 氮肥运筹对钵苗机插优 质食味水稻产量及氮素吸收利用的影响. 作物学报, 2016, 42: 1666-1676.

Hu Q, Xia M, Zhang H C, Cao L Q, Guo B W, Wei H Y, Chen H C, Dai Q G, Huo Z Y, Xu K, Lin C M, Han B F. Effect of nitrogen application regime on yield, nitrogen absorption and utilization of mechanical pot-seedling transplanting rice with good taste quality. Acta Agron Sin, 2016, 42: 1666-1676 (in Chinese with English abstract).

[23] 严田蓉, 李旭毅, 李娜, 蒋明金, 杨志远, 何艳, 王春雨, 王海 月, 马均. 氮肥运筹与栽植方式对杂交籼稻籽粒灌浆及产量 的影响. 中国生态农业学报, 2017, 25: 1485-1494.

Yan T R, Li X Y, Li N, Jiang M J, Yang Z Y, He Y, Wang C Y, Wang H Y, Ma J. Effect of nitrogen management and cultivation method on grain-filling characteristics and grain yield of indica 
hybrid rice. Chin J Eco-Agric, 2017, 25: 1485-1494 (in Chinese with English abstract).

[24] 李贵勇, 何清兰, 朱建宇, 王秋英, 叶进华, 夏琼梅, 龙瑞平, 朱海平, 邓安凤, 杨从党. 不同叶龄期穗肥对籼稻产量及氮肥 利用效率的影响. 中国稻米, 2017, 23(4): 147-150.

Li G Y, He Q L, Zhu J Y, Wang Q Y, Ye J H, Xia Q M, Long R P, Zhu H P, Deng A F, Yang C D. Effects of panicle fertilizers of different leaf ages on japonica rice yield and nitrogen use efficiency. Chin Rice, 2017, 23(4): 147-150 (in Chinese with English abstract).

[25] Fan M S, Lu S H, Jiang R F, Liu X J, Zhang F S. Triangular transplanting pattern and split nitrogen fertilizer application increase rice yield and nitrogen fertilizer recovery. Agron J, 2009, 101: 1421-1425.

[26] 丁艳锋, 赵长华, 王强盛. 穗肥施用时期对水稻氮素利用及产 量的影响. 南京农业大学学报, 2003, 26(4): 5-8.

Ding Y F, Zhao C H, Wang Q S. Effect of application stage of panicle fertilizer on rice grain yield and the utilization of nitrogen. J Nanjing Agric Univ, 2003, 26(4): 5-8 (in Chinese with English abstract).

[27] 霍中洋. 长江中游地区双季早稻超高产形成特征及精确定量 栽培关键技术研究. 扬州大学博士学位论文, 江苏扬州, 2010. Huo Z Y. Study on Super-high Yield Formational Characteristics of Early Rice in the Double-cropping System in the Model Researches of the Yangtze River and Its Key Techniques of Precise and Quantitation Cultivation. PhD Dissertation of Yangzhou University, Yangzhou, Jiangsu, China, 2010 (in Chinese with English abstract).

[28] Williams J F, Mutters R G, Greer C A, Horwath W R. Rice Nutrient Management in California. Richmond, California: Agriculture and Natural Resources, University of California Press, 2010.

[29] Wang H Y, Hu R F, Chen X X, Zhong X H, Zheng Z T, Huang N $\mathrm{R}$, Xue C L. Reduction in nitrogen fertilizer use results in increased rice yields and improved environmental protection. Int $J$ Agric Sustain, 2017, 15: 681-692.

[30] 黄大山, 陈忠平, 程飞虎, 周培建, 林志南, 吴放斌. 不同时 期施用分菜肥对水稻产量及其结构的影响. 江西农业学报, 2014, 26(5): 29-32.

Huang D S, Chen Z P, Cheng F H, Zhou P J, Lin Z N, Wu F B. Effects of applying tillering fertilizer at different times on yield and yield components of rice. Acta Agric Jiangxi, 2014, 26(5):
29-32 (in Chinese with English abstract).

[31] 史鸿儒, 张文忠, 解文孝, 杨庆, 张振宇, 韩亚东, 徐正进, 陈 温福. 不同氮肥施用模式下北方粳型超级稻物质生产特性分 析. 作物学报, 2008, 34: 1985-1993.

Shi H R, Zhang W Z, Xie W X, Yang Q, Zhang Z Y, Han Q D, Xu $\mathrm{Z}$ J, Chen W F. Analysis of matter production characteristics under different nitrogen application patterns of japonica super rice in North China. Acta Agron Sin, 2008, 34: 1985-1993 (in Chinese with English abstract).

[32] 杨海生, 张洪程, 杨连群, 张士永, 戴其根, 霍中洋. 依叶龄 运筹氮肥对优质水稻产量与品质的影响. 中国农业大学学报, 2002, 7(3): 19-26.

Yang H S, Zhang H C, Yang L Q, Zhang S Y, Dai Q G, Huo Z Y. Effects of nitrogen operations according to leaf-age on yield and quality in good-quality rice. J Chin Agric Univ, 2002, 7(3): 19-26 (in Chinese with English abstract).

[33] 林忠成, 李土明, 吴福观, 张洪程, 戴其根, 叶世超, 郭宏文. 基菜肥与穗肥氮比例对双季稻产量和碳氮比的影响. 植物营 养与肥料学报, 2011, 17: 269-275.

Lin Z C, Li T M, Wu F G, Zhang H C, Dai Q G, Ye S C, Guo H W. Effects of nitrogen application on yield and $\mathrm{C} / \mathrm{N}$ of double-cropping rice. J Plant Nutr Fert, 2011, 17: 269-275 (in Chinese with English abstract).

[34] 许轫, 张军, 张洪程, 花劲, 郭保卫, 霍中洋, 戴其根, 魏海燕, 高辉, 周培建, 程飞虎, 黄大山, 陈忠平, 陈国梁. 双季晚粳 稻氮肥精确运筹研究. 植物营养与肥料学报, 2014, 20: 1063-1075.

Xu K, Zhang J, Zhang H C, Hua J, Guo B W, Huo Z Y, Dai Q G, Wei H Y, Gao H, Zhou P J, Cheng F H, Huang D S, Chen Z P, Chen G L. Nitrogen managements of late japonica rice in double-cropping rice area. J Plant Nutr Fert, 2014, 20: 1063-1075 (in Chinese with English abstract).

[35] Wang G H, Dobermann A, Witt C, Sun Q Z, Fu R X. Performance of site-specific nutrient management for irrigated rice in Southeast China. Agron J, 2001, 93: 869-878.

[36] 刘立军, 徐伟, 桑大志, 刘翠莲, 周家麟, 杨建昌. 实地氮肥 管理提高水稻氮肥利用效率. 作物学报, 2006, 32: 987-994.

Liu L J, Xu W, Sang D Z, Liu C L, Zhou J L, Yang J C. Site-specific nitrogen management increases fertilizer-nitrogen use efficiency in rice. Acta Agron Sin, 2006, 32: 987-994 (in Chinese with English abstract). 